\title{
Indium(III)-Catalyzed Synthesis of $N$-Substituted Pyrroles under Solvent-Free Conditions
}

\author{
Jiu-Xi Chen, Miao-Chang Liu, Xiao-Liang Yang, Jin-Chang Ding and Hua-Yue Wu*
}

College of Chemistry and Materials Engineering, Wenzhou University, Wenzhou, 325027, P. R. China

\begin{abstract}
Vários pirróis $N$-substituídos foram sintetizados pela reação de $\gamma$-dicetonas $\left(\mathrm{R}^{1} \mathrm{C}(\mathrm{O})\right.$ $\left.\mathrm{CH}_{2} \mathrm{CH}_{2} \mathrm{C}(\mathrm{O}) \mathrm{R}^{2}: \mathrm{R}^{1}, \mathrm{R}^{2}=\mathrm{Me}, \mathrm{Ph}\right)$ com aminas $\left(\mathrm{RNH}_{2}: \mathrm{R}=\right.$ Alkyl, Aryl, TsNH) ou diaminas (1,6-diaminohexano and 1,2-diaminoetano) na presença de tribrometo de índio, tricloreto de índio ou trifluorometanossulfonato de índio a temperatura ambiente e sem solventes. O protocolo envolve operações simples e os produtos são isolados em excelentes rendimentos (81-98\%).
\end{abstract}

A variety of $N$-substituted pyrroles have been synthesized by reacting $\gamma$-diketones $\left(\mathrm{R}^{1} \mathrm{C}(\mathrm{O})\right.$ $\left.\mathrm{CH}_{2} \mathrm{CH}_{2} \mathrm{C}(\mathrm{O}) \mathrm{R}^{2}: \mathrm{R}^{1}, \mathrm{R}^{2}=\mathrm{Me}, \mathrm{Ph}\right)$ with amines $\left(\mathrm{RNH}_{2}: \mathrm{R}=\mathrm{Alkyl}\right.$, Aryl, TsNH) or diamines (1,6-diaminohexane and 1,2-diaminoethane) in the presence of indium tribromide, indium trichloride or indium trifluoromethanesulfonate at room temperature under solvent-free conditions. The experiment protocol features simple operations, and the products are isolated in high to excellent yields (81-98\%).

Keywords: indium tribromide, indium trichloride, indium trifluoromethanesulfonate, pyrroles, solvent-free conditions

\section{Introduction}

Heterocyclic small molecules play an important role in the search for new therapeutic and drug candidates. ${ }^{1}$ Pyrroles are an important class of heterocyclic compounds and are structural units found in a vast array of natural products, synthetic materials, and bioactive molecules, such as heme, vitamin B12, and cytochromes. ${ }^{2}$ Pyrroles also play crucial roles in nonlinear optical materials as well as in supramolecular chemistry. ${ }^{3}$ Therefore, the enormous number of procedures have been developed for the construction of pyrroles. Classical methods for their preparation include the Knorr, ${ }^{4}$ Hantzsch, ${ }^{5}$ and PaalKnorr condensation reactions. ${ }^{6-16}$ Among these methods, the most commonly used and straightforward approach for the preparation of pyrroles is the direct condensation of $\gamma$-diketones and amines (Paal-Knorr reaction) in the presence of protonic acids such as $\mathrm{AcOH},{ }^{6} p$ - TsOH. ${ }^{7}$ Some improved procedures were reported to utilize Lewis acids such as $\mathrm{Bi}\left(\mathrm{NO}_{3}\right)_{3} \cdot 5 \mathrm{H}_{2} \mathrm{O},{ }^{8} \mathrm{Sc}(\mathrm{OTf})_{3},{ }^{9} \mathrm{SnCl}_{2}{ }^{10}$ and heterogeneous catalyst like montmorillonite (KSF, ${ }^{11}$ $\mathrm{K} 10^{12}$ and $\left.\mathrm{Fe}(\mathrm{III})^{13}\right)$, zirconium compounds. ${ }^{14}$ Recently, this condensation reaction has also been performed under microwave technology ${ }^{15}$ and in ionic liquid medium. ${ }^{16}$

*e-mail: huayuewu@wzu.edu.cn
However, there are some limitations with these methodologies such as prolonged reaction times, ${ }^{11,14,16}$ elevated temperatures, ${ }^{7}$ use of volatile organic solvents, ${ }^{8,11}$ the requirement of excess reagents or catalysts, ${ }^{10}$ moisture sensitive/hazardous catalysts, ${ }^{10}$ use of an additional microwave oven, ${ }^{15}$ use of costly solvent (ionic liquids), ${ }^{16}$ unsatisfactory and yields ${ }^{14}$. Thus, the development of new reagents with great efficiency, environmentally friendly, convenient procedure, and delivery of better yields methods for the synthesis of pyrroles is of great interest.

During the last decade particularly after the review by Cintas, ${ }^{17}$ indium(III) based carbon-carbon bond formation and other organic transformations have gained much attention. In accordance with the recent surge of interest in indium(III) salts has emerged as a promising catalyst for various types of organic transformations. Particularly, indium tribromide ${ }^{18}$ indium trichloride ${ }^{19}$ and indium trifluoromethanesulfonate ${ }^{20}$ as a novel type of water-tolerant green Lewis acid catalyst have received considerable attention for various transformations, because they have advantages of water stability, operational simplicity, recyclability, strong tolerance to oxygen and nitrogencontaining reaction substrates and functional groups, and it can often be used in just catalytic amounts. Recently, Zhang $^{21}$ reported a few examples of $\mathrm{InBr}_{3}$-catalyzed organic 


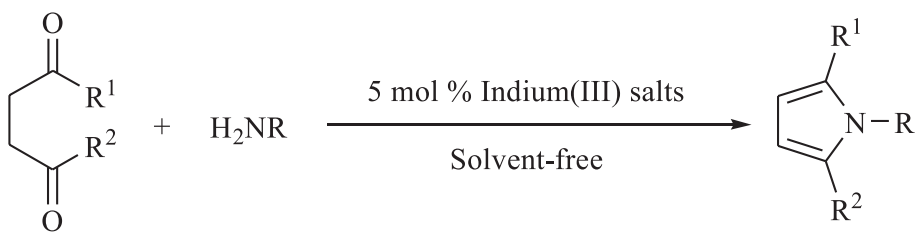

Scheme 1.

reactions. Ghosh and coworkers ${ }^{22}$ reviewed systematically In(OTf $)_{3}$-catalyzed organic syntheses.

In continuation of our interest in Lewis acid-catalyzed and green chemistry organic reactions, ${ }^{23}$ we herein wish to report a simple, practical and efficient method for the synthesis of pyrroles from $\gamma$-diketones and amines using indium(III) salt as catalyst at room temperature under solvent-free conditions (Scheme 1).

\section{Results and Discussion}

Initially, the efficacy of various catalysts was tested in the model reaction under solvent-free condition at room temperature (Table 1). The results showed that $\mathrm{InCl}_{3}, \mathrm{InBr}_{3}$ and $\mathrm{In}(\mathrm{OTf})_{3}$ were superior to other Lewis acids with respect to reaction times and product yields. Entry 1 showed the blank reaction without addition of any catalyst, in this case only $29 \%$ 2,5-dimethyl-1phenyl-1H-pyrrole (3a) was obtained after $24 \mathrm{~h}$. For the sake of comparison, the reaction was carried out using other anhydrous chloride, such as $\mathrm{ZnCl}_{2}, \mathrm{NiCl}_{2}, \mathrm{SrCl}_{2}$ and $\mathrm{CuCl}_{2}$ under solvent free conditions (Entries 2-8). However, these chlorides showed lower activity than $\mathrm{InCl}_{3}$ under same reaction conditions. We examined influence of solvents on the reaction yields. It was found that the reactions gave lower yields of the desired product in solvents such as $\mathrm{CH}_{2} \mathrm{Cl}_{2}, \mathrm{H}_{2} \mathrm{O}, \mathrm{CH}_{3} \mathrm{NO}_{2}$ after prolonged reaction time. (Entries 10-13, 15-18, 20-23). We also checked the reusability of the catalyst by separation and reloading in a new run and found that the catalyst could be reused three times without any decrease in the yield (Entry 19). In light of these good results, subsequent studies were carried out under the following optimized conditions: with $5 \mathrm{~mol} \% \mathrm{InBr}_{3}, \mathrm{InCl}_{3}$ or $\mathrm{In}(\mathrm{OTf})_{3}$ at room temperature under solvent-free conditions.

To evaluate the scope of catalyst's application, various $\gamma$-diketones were treated with amines under the above conditions and the results presented in Table 2. The desired products were characterized by NMR, IR and mass spectrometry and also by comparison with authentic samples.

The Indium(III)-catalyzed reactions were found to be dependent on electronic and steric factors of amines.
Anilines with electron-donating groups gave a high yield (up to $98 \%$ ) due to the increased electron density of the aromatic system (entries 2 and 6). Whereas anilines with strongly electron-withdrawing group $\left(\mathrm{NO}_{2}\right)$ afforded in moderate yield (71\%) after $40 \mathrm{~min}$ (entry 7 ), which showed electronic effect. ortho-substituted anilines whatever the

Table 1. The condensation reaction of acetonylacetone with aniline under various reaction conditions $\mathrm{s}^{\mathrm{a}}$

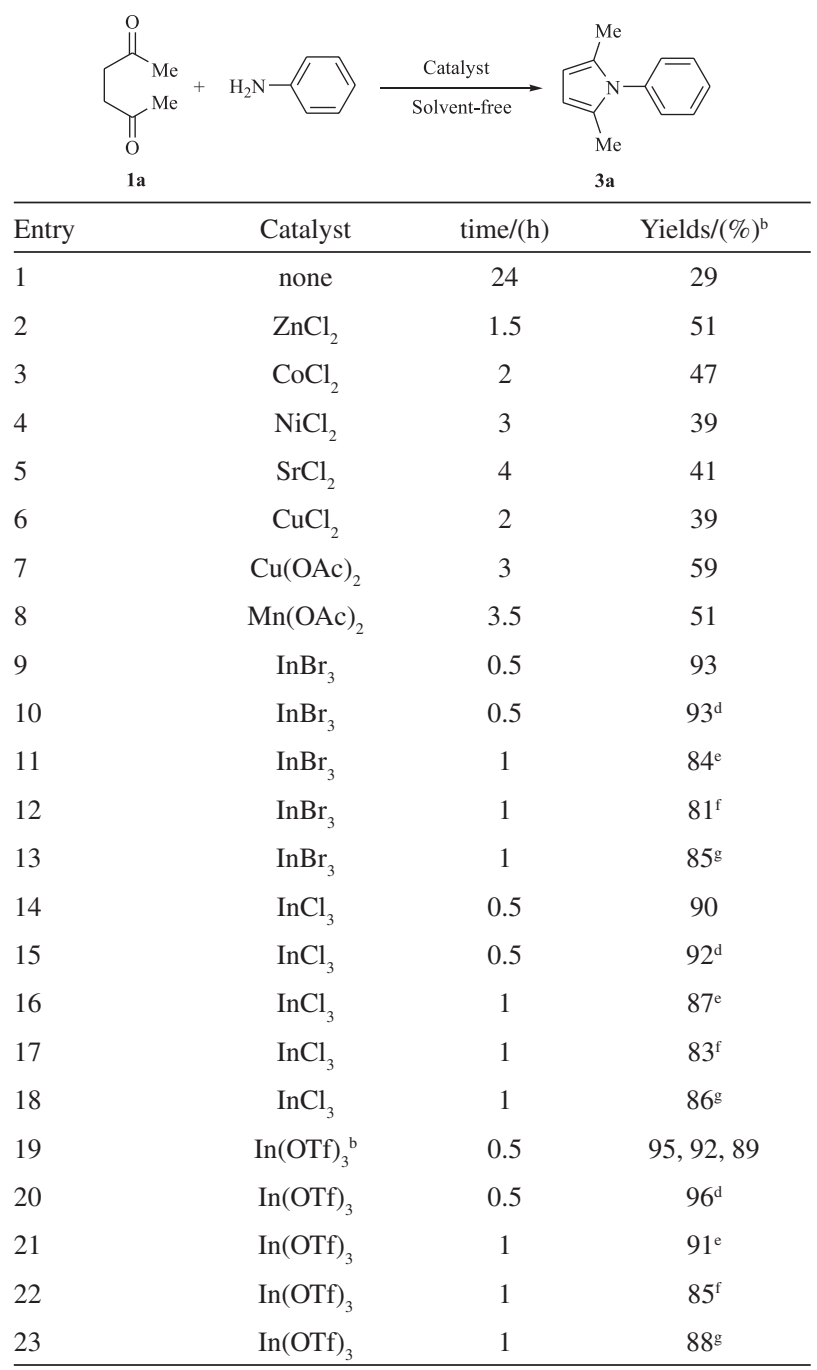

${ }^{\mathrm{a}}$ General reaction conditions: acetonylacetone $(5 \mathrm{mmol})$, aniline $(5 \mathrm{mmol})$, catalyst ( $5 \mathrm{~mol} \%)$, rt. ${ }^{\mathrm{b}} \mathrm{In}(\mathrm{OTf})_{3}$ was reused for three times. ${ }^{\mathrm{I}}$ Isolated yield. ${ }^{\mathrm{d}} 10 \mathrm{~mol} \%$ was used. ${ }^{2} 2 \mathrm{~mL}$ of $\mathrm{CH}_{2} \mathrm{Cl}_{2}$ were used. $2 \mathrm{~mL}$ of $\mathrm{H}_{2} \mathrm{O}$ were used. $\mathrm{g}_{2} \mathrm{~mL}$ of $\mathrm{CH}_{3} \mathrm{NO}_{2}$ were used. 


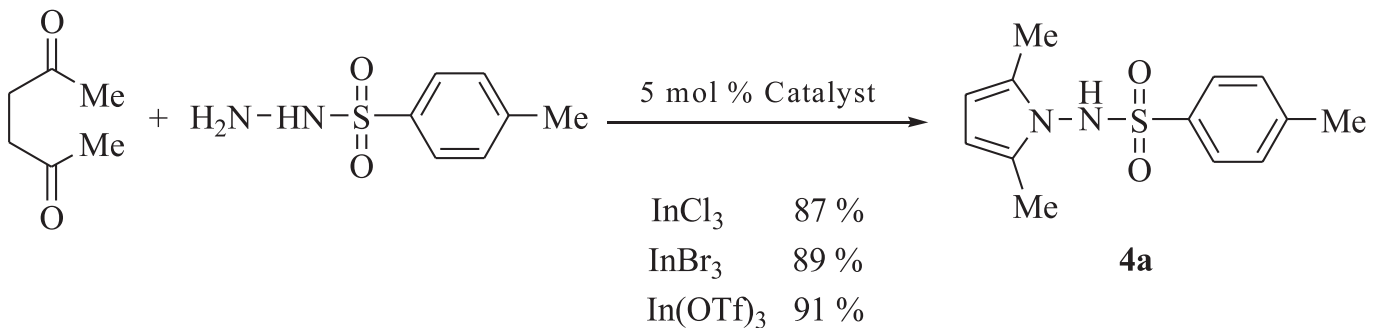

Scheme 2.

character of the substituted groups required longer reaction times. It was revealed that yields were significantly decreased when the sizes of the ortho-substituent groups were large. For example, 2,6-dimethylaniline or 2,6-diisopropylaniline (entries 4-5) were found to be less active and gave mild yields after $1 \mathrm{~h}$. This may be due to the steric hindrance exhibited by the 2,6-diisopropyl and 2,6-dimethyl groups of the aniline towards the approaching acetonylacetone. Moreover, we also examined the condensation reaction of aliphatic amines with acetonylacetone (entries 8-18). The corresponding products $(\mathbf{3 h}-\mathbf{3 r})$ were obtained with excellent yields. In the case of 1,6-diaminohexane or 1,2-diaminoethane, two equivalents of acetonylacetone were used giving products with dipyrryl groups $3 \mathbf{n}$ or $\mathbf{3 o}$ (entries 14-15).

On the other hand, it was found that heterocyclic amines (2-aminopyridine) and less nucleophilic aromatic amine $(\alpha$-naphthalenamine) did not make any difference in this reaction (entries 19 and 20). It is noteworthy that optically active amines were converted into the products (3p-3q) without any racemization or inversion (entries 16 and 17). We also investigated the reaction of various $\gamma$-diketones such as 1,4-diphenylbutane-1,4-dione and 1-phenylpentane-1,4dione with amines. Similarly, the corresponding pyrroles $(3 \mathbf{u}, 3 \mathbf{v})$ were obtained in high yields in the presence of $\mathrm{InCl}_{3}, \mathrm{InBr}_{3}$ or $\mathrm{In}(\mathrm{OTf})_{3}$ at $50{ }^{\circ} \mathrm{C}$.

Finally, we examined the condensation reactions of $\gamma$-diketones with $p$-toluenesulfonylhydrazide (Scheme 2). $N$-(2,5-Dimethyl-1H-pyrrol-1-yl)-4-methylbenzenesulfonamide (4a) were obtained with good yields (87-91\%) when the reaction was performed at $70^{\circ} \mathrm{C}$ in the presence of Indium(III) salts. It is found that this condensation reaction gave 4a in moderate yield (53\%) under solvent-free conditions due to poor solubility of $p$-toluenesulfonylhydrazide in $\gamma$-diketones and the yields showed significant improvement in $\mathrm{CH}_{3} \mathrm{NO}_{2}$. Nevertheless, trace product was obtained in the absence of catalysts, which also further proved that Indium(III) salts do play an important role in this reaction.

The reaction proceeds very cleanly without the formation of any by-products except water. Because the reaction can be performed using a solvent-free procedure, at the end of the reaction, the crude mixture can be directly charged on a chromatographic column to obtain the pure product, avoiding any tedious work up.

In summary, we have developed a facile and efficient method for the synthesis of $N$-substituted pyrroles using Indium(III) salts. The advantages of the method include (i) absence of a solvent, (ii) short reaction times, (iii) high yields, (iv) no work-up, and (v) use of trace amounts of a catalyst that could be recovered and re-used.

\section{Experimental}

Melting points were recorded on Digital Melting Point Apparatus WRS-1B and are uncorrected. ${ }^{1} \mathrm{H}$ NMR and ${ }^{13} \mathrm{C}$ NMR spectra were taken with a VARIAN Mercury plus-400 instrument using $\mathrm{CDCl}_{3}$ as the solvent with tetramethylsilane (TMS) as an internal standard at room temperature. Chemical shifts are given in $\delta$ relative to TMS, the coupling constants $J$ are given in Hz. IR spectra was recorded on a AVATAR 370 FI-Infrared Spectrophotometer. Mass spectra were recorded on Finnigan Trace DSQ (EI, CI) or Thermo Finnigan LCQ-Advantage (ESI).

\section{Typical experimental procedure for the synthesis of} 2,5-dimethyl-1-phenyl-1H-pyrrole (3a)

A mixture of acetonylacetone $(570 \mathrm{mg}, 5 \mathrm{mmol}$ ) and aniline (465 mg, $5 \mathrm{mmol}$ ) was stirred in the presence of $\mathrm{InCl}_{3}(55 \mathrm{mg}, 5 \mathrm{a} \mathrm{mol} \%), \operatorname{InBr}_{3}(89 \mathrm{mg}, 5 \mathrm{~mol} \%)$ or In(OTf) (141 mg, $5 \mathrm{~mol} \%$ ) at room temperature for $30 \mathrm{~min}$. After completion of the reaction (TLC), the crude products were separated by column chromatography on $\mathrm{Et}_{3} \mathrm{~N}$ pre-treated silica gel using petroleum ether/EtOAc (12:1 to 5:1) as eluent to afford a pure product $\mathbf{3 a}$ in $90 \%, 93 \%$ or $94 \%$ yields, respectively. The spectral and analytical data of some representative compounds are given below.

\section{2,5-dimethyl-1-phenyl-1H-pyrrole $(3 \boldsymbol{a})^{13}$}

A white solid, mp 50.1-50.3 ${ }^{\circ} \mathrm{C}\left(46-47^{\circ} \mathrm{C}\right) ; \mathrm{IR} v_{\max } / \mathrm{cm}^{-1}$ 3060, 2922, 1599, 1520, 1498, 1403, 1321 (film); ${ }^{1} \mathrm{H}$ NMR 
Table 2. Indium(III)-catalyzed synthesis of pyrroles under solvent-free conditions ${ }^{\mathrm{a}}$

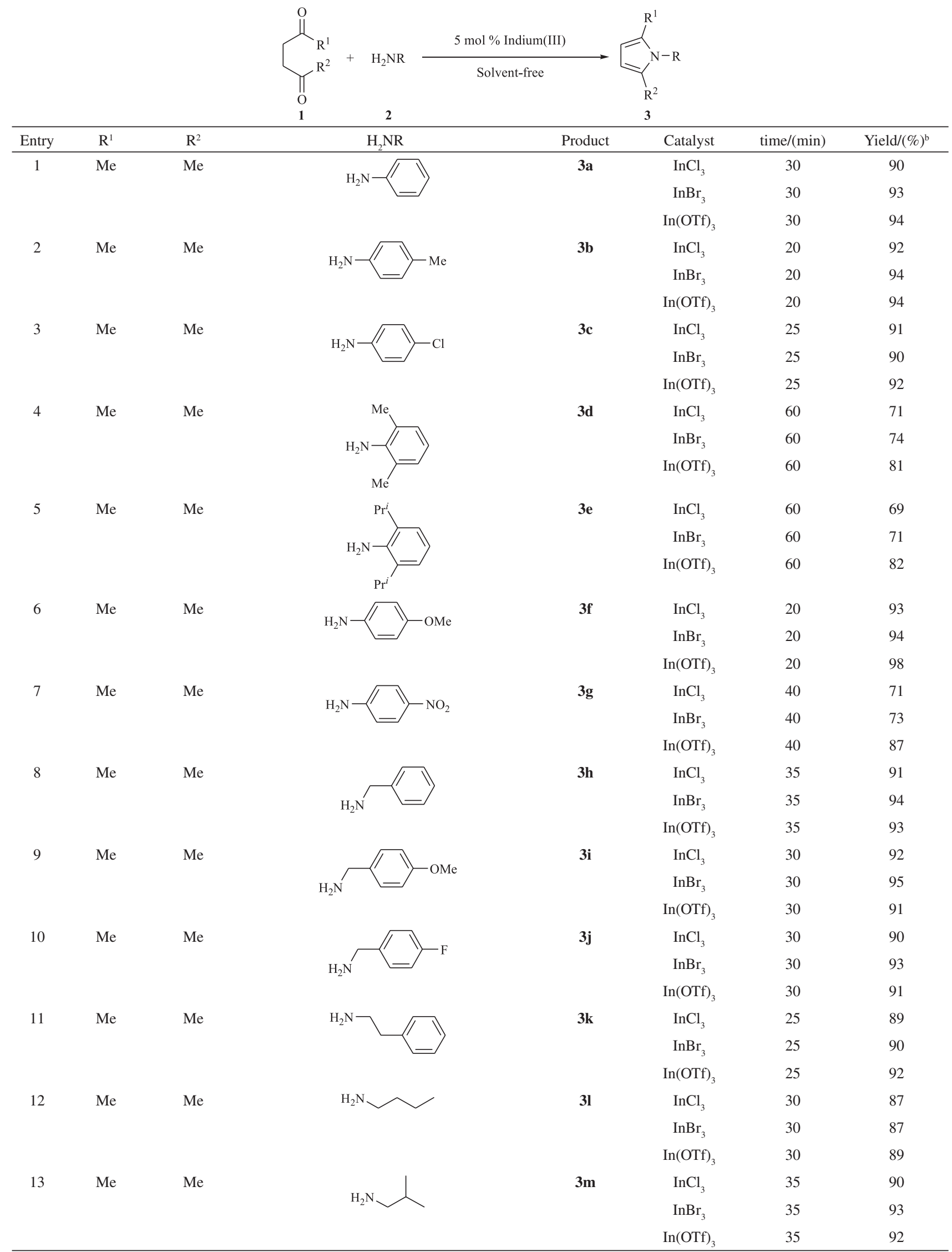


Table 2. continuation

\begin{tabular}{|c|c|c|c|c|c|c|c|}
\hline Entry & $\mathrm{R}^{1}$ & $\mathrm{R}^{2}$ & $\mathrm{H}_{2} \mathrm{NR}$ & Product & Catalyst & time/(min) & Yield $/(\%)^{\mathrm{b}}$ \\
\hline \multirow[t]{3}{*}{14} & \multirow[t]{3}{*}{$\mathrm{Me}$} & \multirow[t]{3}{*}{$\mathrm{Me}$} & & \multirow[t]{3}{*}{$3 n$} & $\mathrm{InCl}_{3}$ & 40 & 88 \\
\hline & & & & & $\mathrm{InBr}_{3}$ & 40 & 87 \\
\hline & & & & & $\operatorname{In}(\mathrm{OTf})_{3}$ & 40 & 90 \\
\hline \multirow[t]{3}{*}{15} & \multirow[t]{3}{*}{$\mathrm{Me}$} & \multirow[t]{3}{*}{$\mathrm{Me}$} & & \multirow[t]{3}{*}{30} & $\mathrm{InCl}_{3}$ & 45 & 88 \\
\hline & & & & & $\mathrm{InBr}_{3}$ & 45 & 87 \\
\hline & & & & & $\operatorname{In}(\mathrm{OTf})_{3}$ & 45 & 92 \\
\hline \multirow[t]{3}{*}{16} & \multirow[t]{3}{*}{$\mathrm{Me}$} & \multirow[t]{3}{*}{$\mathrm{Me}$} & & \multirow[t]{3}{*}{$3 p$} & $\mathrm{InCl}_{3}$ & 40 & 86 \\
\hline & & & & & $\mathrm{InBr}_{3}$ & 40 & 90 \\
\hline & & & & & $\operatorname{In}(\mathrm{OTf})_{3}$ & 40 & 89 \\
\hline \multirow[t]{3}{*}{17} & \multirow[t]{3}{*}{$\mathrm{Me}$} & \multirow[t]{3}{*}{$\mathrm{Me}$} & & \multirow[t]{3}{*}{$3 q$} & $\mathrm{InCl}_{3}$ & 35 & 87 \\
\hline & & & & & $\mathrm{InBr}_{3}$ & 35 & 89 \\
\hline & & & & & $\operatorname{In}(\mathrm{OTf})_{3}$ & 35 & 93 \\
\hline \multirow[t]{3}{*}{18} & \multirow[t]{3}{*}{$\mathrm{Me}$} & \multirow[t]{3}{*}{$\mathrm{Me}$} & & \multirow[t]{3}{*}{$3 r$} & $\mathrm{InCl}_{3}$ & 25 & 90 \\
\hline & & & & & $\mathrm{InBr}_{3}$ & 25 & 89 \\
\hline & & & & & $\operatorname{In}(\mathrm{OTf})_{3}$ & 25 & 93 \\
\hline \multirow[t]{3}{*}{19} & \multirow[t]{3}{*}{$\mathrm{Me}$} & \multirow[t]{3}{*}{$\mathrm{Me}$} & & \multirow[t]{3}{*}{$3 \mathrm{~s}$} & $\mathrm{InCl}_{3}$ & 40 & 89 \\
\hline & & & & & $\mathrm{InBr}_{3}$ & 40 & 92 \\
\hline & & & & & $\operatorname{In}(\mathrm{OTf})_{3}$ & 40 & 90 \\
\hline \multirow[t]{3}{*}{$20^{c}$} & \multirow[t]{3}{*}{$\mathrm{Me}$} & \multirow[t]{3}{*}{$\mathrm{Me}$} & & \multirow[t]{3}{*}{$3 t$} & $\mathrm{InCl}_{3}$ & 35 & 88 \\
\hline & & & & & $\mathrm{InBr}_{3}$ & 35 & 90 \\
\hline & & & & & $\operatorname{In}(\mathrm{OTf})_{3}$ & 35 & 91 \\
\hline \multirow[t]{3}{*}{$21^{c}$} & \multirow[t]{3}{*}{$\mathrm{Ph}$} & \multirow[t]{3}{*}{$\mathrm{Me}$} & & $3 u$ & $\mathrm{InCl}_{3}$ & 35 & 87 \\
\hline & & & & & $\mathrm{InBr}_{3}$ & 35 & 87 \\
\hline & & & & & $\operatorname{In}(\mathrm{OTf})_{3}$ & 35 & 90 \\
\hline $22^{c}$ & $\mathrm{Ph}$ & $\mathrm{Ph}$ & & $3 v$ & $\mathrm{InCl}_{3}$ & 40 & 88 \\
\hline & & & & & $\mathrm{InBr}_{3}$ & 40 & 86 \\
\hline & & & & & $\operatorname{In}(\mathrm{OTf})_{3}$ & 40 & 89 \\
\hline
\end{tabular}

${ }^{\mathrm{a}}$ General reaction conditions: $\gamma$-diketones $(5 \mathrm{mmol})$, aniline $(5 \mathrm{mmol})$, catalyst $(5 \mathrm{~mol} \%)$, rt. ${ }^{\mathrm{b}}$ Isolated yield. ${ }^{\mathrm{c}}$ The reaction was carried out at $50{ }^{\circ} \mathrm{C}$.

$\left(400 \mathrm{MHz}, \mathrm{CDCl}_{3}\right) \delta$ 7.49-7.41 (m, 3H, ArH), 7.27-7.22 (m, 2H, ArH), 5.92 (s, 2H, pyrrole), $2.05\left(\mathrm{~s}, 6 \mathrm{H}, \mathrm{CH}_{3}\right) .{ }^{13} \mathrm{C}$ NMR $\left(100 \mathrm{MHz}, \mathrm{CDCl}_{3}\right) \delta 139.9,129.0,128.8,127.6$, 105.6, 13.0; MS (EI): $m / z$ (\%) 171 (100) $\left[\mathrm{M}^{+}\right], 156$ (8). Compounds $\mathbf{3 b - 3 m}$ and $\mathbf{3 p - 3 v}$ were obtained following a similar procedure.

\section{2,5-dimethyl-1-((R)-1-phenylethyl)-1H-pyrrole $(\mathbf{3 p})^{24}$}

A colorless oil, $[\alpha]_{\mathrm{D}}{ }^{20}:+42.95\left(c 0.098, \mathrm{CH}_{3} \mathrm{OH}\right)$; IR $v_{\max } / \mathrm{cm}^{-1} 3088,2975,2926,1519,1496,1448,1395$, 1293 (neat); ${ }^{1} \mathrm{H}$ NMR (400 MHz, $\mathrm{CDCl}_{3}$ ) $\delta 7.27$ (t, J 7.2 $\mathrm{Hz}, 2 \mathrm{H}, \mathrm{ArH}), 7.20$ (t, J 7.2 Hz, 2H, ArH), 7.02 (d, J 7.2 $\mathrm{Hz}, 2 \mathrm{H}, \mathrm{ArH}), 5.80$ (s, $2 \mathrm{H}$, pyrrole), 5.45 (q, J 7.2 Hz, 1H, $\left.\mathrm{CHCH}_{3}\right), 2.06\left(\mathrm{~s}, 6 \mathrm{H}, \mathrm{CH}_{3}\right), 1.83\left(\mathrm{~d}, J 7.2 \mathrm{~Hz}, 3 \mathrm{H}, \mathrm{CHCH}_{3}\right)$. ${ }^{13} \mathrm{C} \mathrm{NMR}\left(100 \mathrm{MHz} \mathrm{CDCl}_{3}\right) \delta 142.3,128.3,128.1,126.7$,
125.9, 106.0, 52.3, 19.2, 13.7; MS (EI): $m / z$ (\%) 200 (52) $[\mathrm{M}+1]^{+}, 199$ (100), 124 (10), 96 (35).

\section{1-(furan-2-ylmethyl)-2,5-dimethyl-1H-pyrrole $(3 \boldsymbol{r})^{9}$}

A colorless oil, IR $v_{\max } / \mathrm{cm}^{-1} 3109,2975,2931,1577$, 1521, 1505, 1442, 1406, 1343, 1296, 1216, 1147 (neat); ${ }^{1} \mathrm{H}$ $\mathrm{NMR}\left(400 \mathrm{MHz}, \mathrm{CDCl}_{3}\right) \delta 7.34$ (s, 1H, furan), 6.31 (s, $1 \mathrm{H}$, furan), 6.05 (s, 1H, furan), $5.83(\mathrm{~s}, 2 \mathrm{H}$, pyrrole), 4.93 (s, $\left.2 \mathrm{H}, \mathrm{CH}_{2}\right), 2.30\left(\mathrm{~s}, 6 \mathrm{H}, \mathrm{CH}_{3}\right) \cdot{ }^{13} \mathrm{C} \mathrm{NMR}\left(100 \mathrm{MHz}, \mathrm{CDCl}_{3}\right)$ $\delta 151.3,142.1,127.9,110.2,106.9,105.3,40.5,12.4$; MS (EI): $m / z(\%) 176(43)[\mathrm{M}+1]^{+}, 175(100)\left[\mathrm{M}^{+}\right], 81(13)$.

\section{2-(2,5-dimethyl-1H-pyrrol-1-yl)pyridine $(3 t)^{14}$}

A white solid, mp 33.2-33.9 ${ }^{\circ} \mathrm{C}$; IR $v_{\max } / \mathrm{cm}^{-1} 3055$, 
2922, 1587, 1525, 1473, 1438, 1397, 1325, 1285, 1234 (film); ${ }^{1} \mathrm{H}$ NMR (400 MHz, $\mathrm{CDCl}_{3}$ ) $\delta 8.62(\mathrm{~d}, J 4.4 \mathrm{~Hz}$, $1 \mathrm{H}$, pyridine), 7.84 (t, $J 7.8 \mathrm{~Hz}, 1 \mathrm{H}$, pyridine), 7.31 (t, $J 6.4$ $\mathrm{Hz}, 1 \mathrm{H}$, pyridine), 7.26-7.22 (m, 1H, pyridine), 5.91 (s, 2H, pyrrole), 2.13 (s, 6H, $\left.\left.\mathrm{CH}_{3}\right) .{ }^{13} \mathrm{C} \mathrm{NMR} \mathrm{(100} \mathrm{MHz,} \mathrm{CDCl}_{3}\right) \delta$ 152.0, 149.2, 138.0, 128.6, 122.3, 122.0, 106.9, 13.1; MS (EI): $m / z(\%) 173(25)[\mathrm{M}+1]^{+}, 172(100)\left[\mathrm{M}^{+}\right], 171(47)$, 157 (25), 94 (13).

1-benzyl-2-methyl-5-phenyl-1H-pyrrole $(3 \boldsymbol{u})^{25}$

A colorless oil, ${ }^{1} \mathrm{H}$ NMR $\left(400 \mathrm{MHz}, \mathrm{CDCl}_{3}\right) \delta 7.30-7.20$ (m, 8H, ArH), 6.92 (d, J 7.2 Hz, 2H, ArH), 6.23 (d, J 2.8 $\mathrm{Hz}, 1 \mathrm{H}$, pyrrole), 6.05 (s, 1H, pyrrole), 5.11 (s, 2H), 2.12 $(\mathrm{s}, 3 \mathrm{H}) .{ }^{13} \mathrm{C} \mathrm{NMR}\left(100 \mathrm{MHz}, \mathrm{CDCl}_{3}\right) \delta 138.9,134.6$, 133.7, 130.4, 128.7, 128.6, 128.3, 126.9, 126.6, 125.6, 107.9, 107.1, 47.6, 12.5; MS (ESI): $m / z$ (\%) 248.2 (100) $[\mathrm{M}+1]^{+}$.

\section{1,2,5-triphenyl-1H-pyrrole $(3 v)^{26}$}

A white solid, mp $230-232{ }^{\circ} \mathrm{C} ;{ }^{1} \mathrm{H}$ NMR (400 MHz, $\left.\mathrm{CDCl}_{3}\right) \delta$ 7.26-7.03 (m, 15H, ArH), 6.5 (s, 2H, pyrrole). ${ }^{13} \mathrm{C} \mathrm{NMR}\left(100 \mathrm{MHz}, \mathrm{CDCl}_{3}\right) \delta 138.9,135.8,133.2,128.9$, 128.7, 127.8, 127.2 (2C), 126.20, 109.9; MS (ESI): $\mathrm{m} / \mathrm{z}(\%)$ $296.1(100)[\mathrm{M}+1]^{+}$.

Typical experimental procedure for the synthesis of 1,6-bis(2,5-dimethyl-1H-pyrrol-1-yl)hexane (3n)

A mixture of acetonylacetone $(1.14 \mathrm{~g}, 10 \mathrm{mmol})$ and 1,6-diaminohexane $(580 \mathrm{mg}, 5 \mathrm{mmol})$ was stirred in the presence of $\mathrm{InCl}_{3}(55 \mathrm{mg}, 5 \mathrm{~mol} \%), \mathrm{InBr}_{3}(89 \mathrm{mg}, 5 \mathrm{~mol} \%$ ) or $\operatorname{In}(\mathrm{OTf})_{3}(141 \mathrm{mg}, 5 \mathrm{~mol} \%)$ at room temperature for $45 \mathrm{~min}$. After completion of the reaction (TLC), the crude products were separated by column chromatography on $\mathrm{Et}_{3} \mathrm{~N}$ pre-treated silica gel using petroleum ether/EtOAc (15:1 to 5:1) as eluent to afford a pure product $\mathbf{3 n}$ in 88 , 87 or $90 \%$ yields. The spectral and analytical data of some representative compounds are given below.

\section{1,6-bis(2,5-dimethyl-1H-pyrrol-1-yl)hexane $(3 n)^{27}$}

A white solid, mp 102.8-103.0 ${ }^{\circ} \mathrm{C}$; IR $v_{\max } / \mathrm{cm}^{-1} 3103$, 3093, 2969, 2924, 1518, 1475, 1410, 1373, 1301 (film); ${ }^{1} \mathrm{H}$ NMR (400 MHz, $\mathrm{CDCl}_{3}$ ) $\delta 5.76$ (s, 4H, pyrrole), 3.70 $(\mathrm{t}, J 7.6 \mathrm{~Hz}, 4 \mathrm{H}), 2.20\left(\mathrm{~s}, 12 \mathrm{H}, \mathrm{CH}_{3}\right), 1.65-1.58(\mathrm{~m}, 4 \mathrm{H})$, 1.38-1.34 (m, 4H). $\left.{ }^{13} \mathrm{C} \mathrm{NMR} \mathrm{(100} \mathrm{MHz,} \mathrm{CDCl}_{3}\right) \delta 127.2$, 105.0, 43.5, 30.9, 26.7, 12.5; MS (EI): $\mathrm{m} / \mathrm{z}$ (\%) 272 (100) $\left[\mathrm{M}^{+}\right], 257$ (8), 164 (38), 108 (30), 94 (18). Compound 3o was obtained following a similar procedure.
$N$-(2,5-dimethyl-1H-pyrrol-1-yl)-4-methylbenzenesulfonamide $(\mathbf{4 a})^{28}$

A white solid, mp $143-144{ }^{\circ} \mathrm{C} ;{ }^{1} \mathrm{H}$ NMR $(400 \mathrm{MHz}$, $\left.\mathrm{CDCl}_{3}\right) \delta 7.68(\mathrm{~d}, J 8.0 \mathrm{~Hz}, 2 \mathrm{H}, \mathrm{ArH}), 7.31(\mathrm{~d}, J 8.0 \mathrm{~Hz}, 2 \mathrm{H}$, ArH), 7.07 (s, 1H, NH), 5.69 (s, 2H, pyrrole), 2.45 (s, 3H, $\left.\mathrm{ArCH}_{3}\right), 1.82\left(\mathrm{~s}, 3 \mathrm{H}, \mathrm{CH}_{3}\right) \cdot{ }^{13} \mathrm{C} \mathrm{NMR}\left(100 \mathrm{MHz}, \mathrm{CDCl}_{3}\right)$ $\delta$ 145.0, 135.3, 129.9, 128.9, 128.4, 104.9, 21.7, 11.2; MS (ESI): $m / z(\%) 265.2(100)[\mathrm{M}+1]^{+}$.

\section{Acknowledgments}

We are grateful to the Natural Science Foundation of Zhejiang Province (No. Y405015 and Y405113) for financial support.

\section{Supplementary Information}

Supplementary data are available free of charge at http:// jbcs.sbq.org.br, as PDF file.

\section{References}

1. Couladouros, E. A.; Strongilos, A. T.; Angew. Chem. Int. Ed. 2002, 41, 3677.

2. Jones, R. A.; Bean, G. P.; The Chemistry of Pyrroles, Academic: London, 1977; Joule, J. A.; Mills, K.; Heterocyclic Chemistry, $4^{\text {th }}$ ed.; Blackwell Science: Oxford, UK, 2000; Bellina, F.; Rossi, R.; Tetrahedron 2006, 62, 7213; Butler, M. S.; J. Nat. Prod. 2004, 67, 2141; Hoffmann, H.; Lindel, T.; Synthesis 2003, 1753.

3. Lehn, J. M.; Supramolecular Chemistry: Concepts and Perspectives, VCH: Weinheim, Germany, 1995.

4. Knorr, L.; Ber. 1884, 17, 1635; Kleinspehn, G. G.; J. Am. Chem. Soc. 1955, 77, 1546; Hamby, J. M.; Hodges, J. C.; Heterocycles 1993, 35, 843; Shiner, C. M.; Lash, T. D.; Tetrahedron 2005, 61,11628 .

5. Hantzsch, A.; Ber. 1890, 23, 1474; Trautwein, A. W.; Süßmuth, R. D.; Jung, G.; Bioorg. Med. Chem. Lett. 1998, 8, 2381; Ferreira, V. F.; De Souza, M. C. B. V.; Cunha, A. C.; Pereira, L. O. R.; Ferreira, M. L. G.; Org. Prep. Proced. Int. 2002, 33, 411; Matiychuk, V. S.; Martyak, R. L.; Obushak, N. D.; Ostapiuk, Y. V.; Pidlypnyi, N. I.; Chem. Heterocycl. Compd. 2004, 40, 1218.

6. Knorr, L.; Ber. 1884, 17, 2863; Paal, C.; Ber. 1884, 17, 2756; Hori, I.; Igarashi, M.; Bull. Chem. Soc. Jpn. 1971, 44, 2856; Braun, R. U.; Zeitler, K.; Mueller, T. J. J.; Org. Lett. 2001, 3, 3297.

7. Bharadwaj, A. R.; Scheidt, K. A.; Org. Lett. 2004, 6, 2465; Tracey, M. R.; Hsung, R. P.; Lambeth, R. H.; Synthesis 2004, 918.

8. Banik, B. K.; Banik, I.; Renteria, M.; Dasgupta, S. K.; Tetrahedron Lett. 2005, 46, 2643. 
9. Chen, J.; Wu, H.; Zheng, Z.; Jin, C.; Zhang, X.; Su, W.; Tetrahedron Lett. 2006, 47, 5383.

10. Arumugam, P.; Perumal, P. T.; Chem. Lett. 2006, 35, 632.

11. Banik, B. K.; Samajdar, S.; Banik, I.; J. Org. Chem. 2004, 69, 213; Samajdar, S.; Becker, F. F.; Banik, B. K.; Heterocycles 2001, 55, 1019.

12. Texier-Boullet, F.; Klein, B.; Hamelin, J.; Synthesis 1986, 409.

13. Wang, B.; Kang, Y. R.; Yang, T.; Yang, L. M.; Synth. Commun. 2005, 35, 1051.

14. Curini, M.; Montanari, F.; Rosati, O.; Lioy, E.; Margarita, R.; Tetrahedron Lett. 2003, 44, 3923.

15. Werner, S.; Iyer, P. S.; Synlett 2005, 1405; Minetto, G.; Raveglia, L. F.; Sega, A.; Taddei, M.; Eur. J. Org. Chem. 2005, 70, 5277; Danks, T. N.; Tetrahedron Lett. 1999, 40, 3957; Minetto, G.; Raveglia, L. F.; Taddei, M.; Org. Lett. 2004, 6, 389; Rao, H. S. P.; Jothilingam, S.; Scheeren, H. W.; Tetrahedron 2004, 60, 1625; Rao, H. S. P.; Jothilingam, S.; Tetrahedron Lett. 2001, 42, 6595; Abid, M.; Landge, S. M.; Torok, B.; Org. Prep. Proced. Int. 2006, 38, 495.

16. Yadav, J. S.; Reddy, B. V. S.; Eeshwaraiah, B.; Gupta, M. K.; Tetrahedron Lett. 2004, 45, 5873; Wang, B.; Gu, Y. L.; Luo, C.; Yang, T.; Yang, L. M.; Suo, J. S.; Tetrahedron Lett. 2004, 45, 3417.

17. Cintas, P.; Synlett 1995, 1087.

18. Chen, C.; Liu, L.; Yang, D.; Wang, D.; Chen, Y.; Synlett 2005, 2047; Miura, K.; Fujisawa, N.; Toyohara, S.; Hosomi, A.; Synlett 2006, 1883; Angell, P.; Blazecka, P. G.; Lovdahl, M.; Zhang, J.; J. Org. Chem. 2007, 72, 6606; Manian, R.; Durga, R. S.; Jayashankaran, J.; Raghunathan, R.; Tetrahedron Lett. 2007, $48,4139$.
19. Smith, B. M.; Skellam, E. J.; Oxley, S. J.; Graham, A. E.; Org. Biomol. Chem. 2007, 5, 1979; Vicennati, P.; Cozzi, P. G.; Eur. J. Org. Chem. 2007, 2248; Yadav, J. S.; Reddy, B. V. S.; Basak, A. K.; Baishya, G.; Narsaiah, A. V.; Synthesis 2006, 3831; Zhang, Z.; Yin, L.; Wang, Y. M.; Adv. Synth. Catal. 2006, 348, 184.

20. Pellicciari, R.; Amori, L.; Kuznetsova, N.; Zlotsky, S. Gioiello, A.; Tetrahedron Lett. 2007, 48, 4911; Weiewer, M.; Chaminade, X.; Bayon, J. C.; Dunach, E.; Eur. J. Org. Chem. 2007, 2464; Saito, A.; Taguchi, T.; Tetrahedron 2005, 61, 7087; Weïwer, M.; Duñach, E.; Tetrahedron Lett. 2006, 47, 287.

21. Zhang, Z.; Synlett 2005, 711.

22. Ghosh, R.; Maiti, S.; J. Mol. Catal. A, Chem. 2006, 264, 1.

23. Chen, J. X.; Wu, H. Y.; Jin, C.; Zhang, X. X.; Xie, Y. Y.; Su, W. K.; Green Chem. 2006, 8, 330; Chen, J. X.; Su, W. K.; Wu, H. Y.; Liu, M. C.; Jin, C.; Green Chem. 2007, 9, 972; Su, W. K.; Chen, J. X.; Wu, H. Y.; Jin, C.; J. Org. Chem. 2007, 72, 4524; Chen, X. A.; Zhang, C. F.; Wu, H. Y.; Yu, X. C.; Su, W. K.; Cheng, J.; Synthesis 2007, 3233.

24. Nishiyama, T.; Mizuno, K.; Otsuji, Y.; Inoue, H.; Chem. Lett. 1994, 2227.

25. Muthusamy, S.; Babu, S. A.; Gunanathan, C.; Tetrahedron 2002, $58,7897$.

26. Periasamy, M.; Srinivas, G.; Bharath, P.; J. Org. Chem. 1999, 64, 4204.

27. Buu-Hoi, N. P.; J. Chem. Soc. 1949, 2882.

28. Kermack, W. O.; Weatherhead, A. P.; Walsh, E. O.; King, F. E.; King, T. J.; J. Chem. Soc. 1942, 726.

Received: April 11, 2007 Web Release Date: April 18, 2008 


\section{Indium(III)-catalyzed Synthesis of $\mathrm{N}$-Substituted Pyrroles under Solvent-free Conditions}

Jiu-Xi Chen, Miao-Chang Liu, Xiao-Liang Yang, Jin-Chang Ding and Hua-Yue Wu*

College of Chemistry and Materials Engineering, Wenzhou University, Wenzhou, 325027, P. R. China

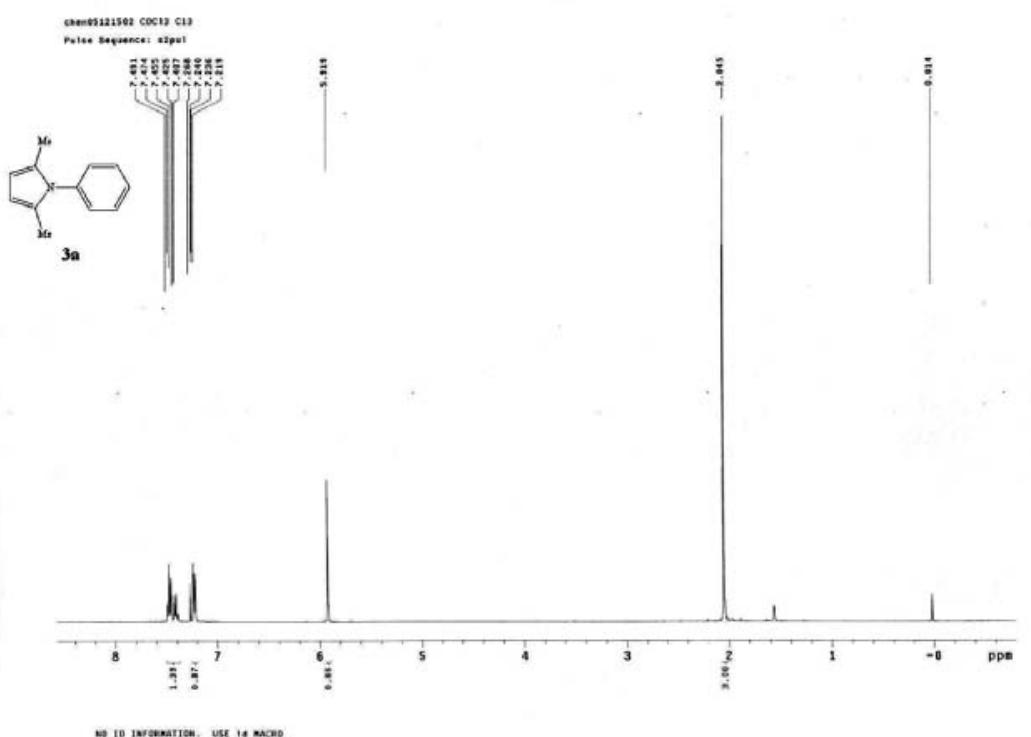

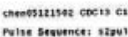
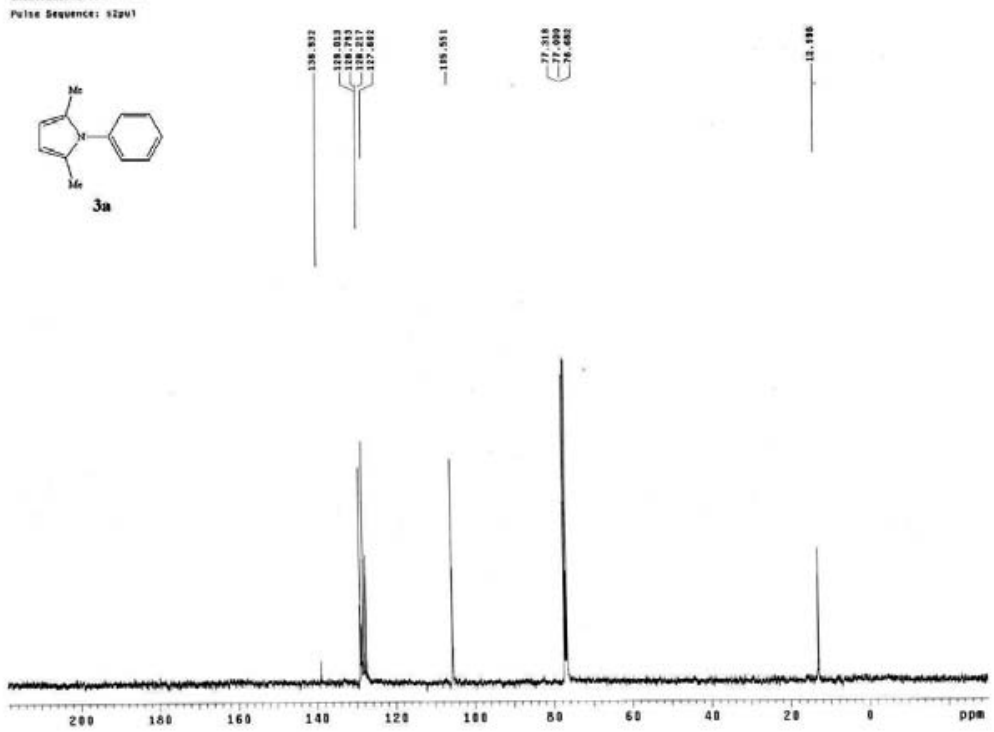

Figure S1. ${ }^{1} \mathrm{H}$ NMR of $\mathbf{3 a}\left(400 \mathrm{MHz}, \mathrm{CDCl}_{3}\right)$ and ${ }^{13} \mathrm{C} \mathrm{NMR}$ of $\mathbf{3 a}\left(100 \mathrm{MHz}, \mathrm{CDCl}_{3}\right)$. 


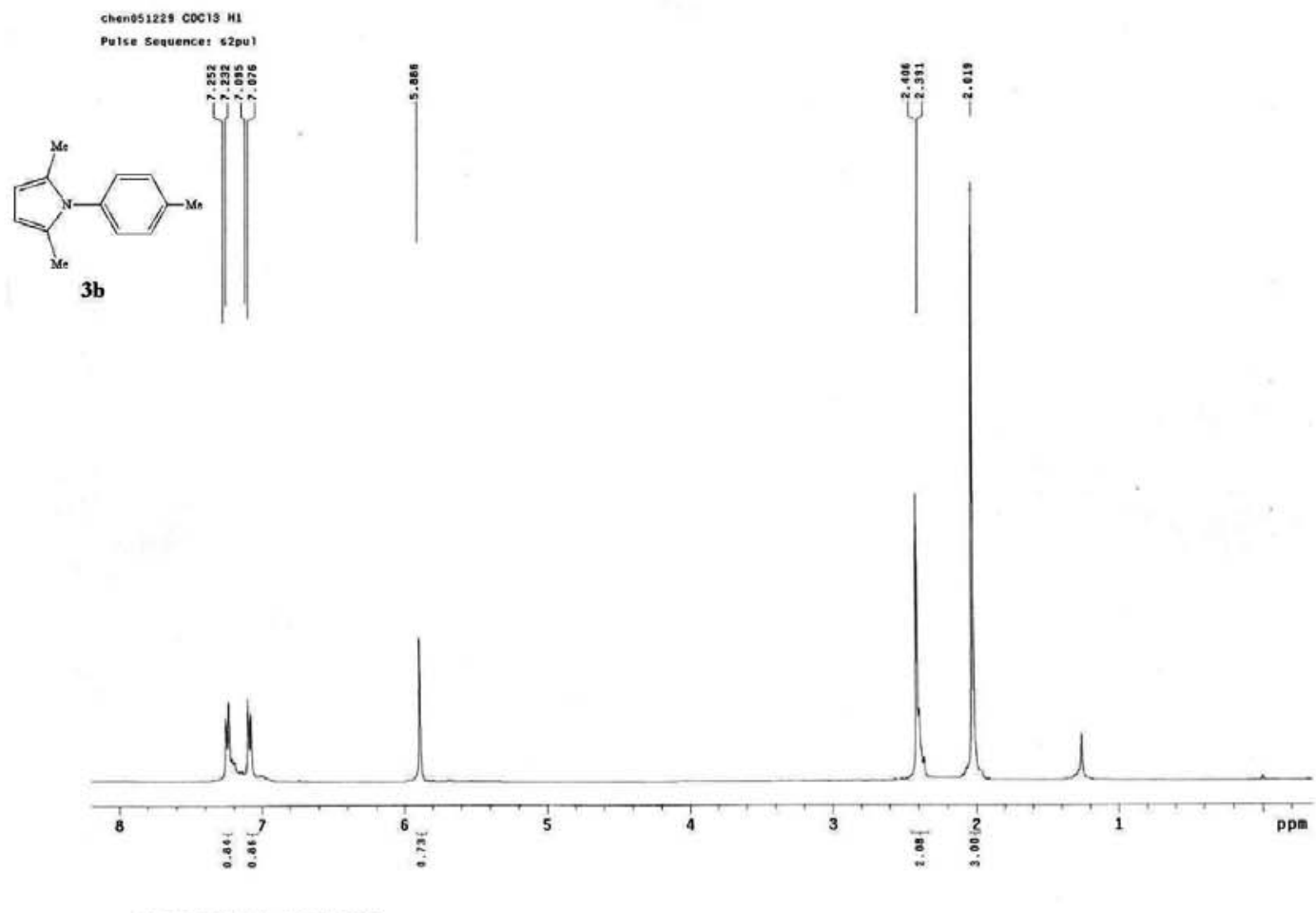

mo to ImroRmation. USt it Macko

chenosize, cocis c1s Pulse Sequence: s2pul
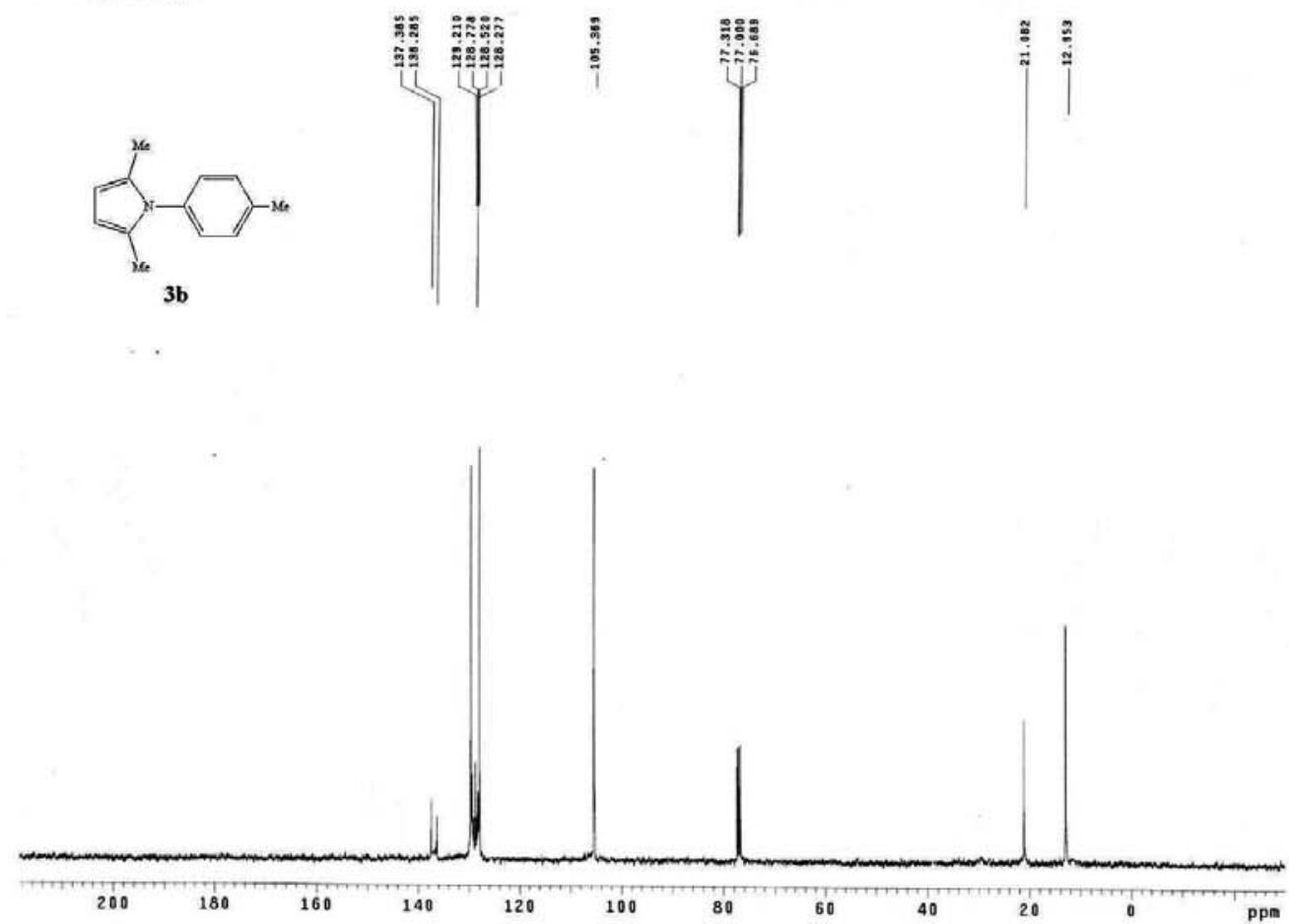

Figure S2. ${ }^{1} \mathrm{H} \mathrm{NMR}$ of $\mathbf{3 b}\left(400 \mathrm{MHz}, \mathrm{CDCl}_{3}\right)$ and ${ }^{13} \mathrm{C} \mathrm{NMR}$ of $\mathbf{3 b}\left(100 \mathrm{MHz}, \mathrm{CDCl}_{3}\right)$. 


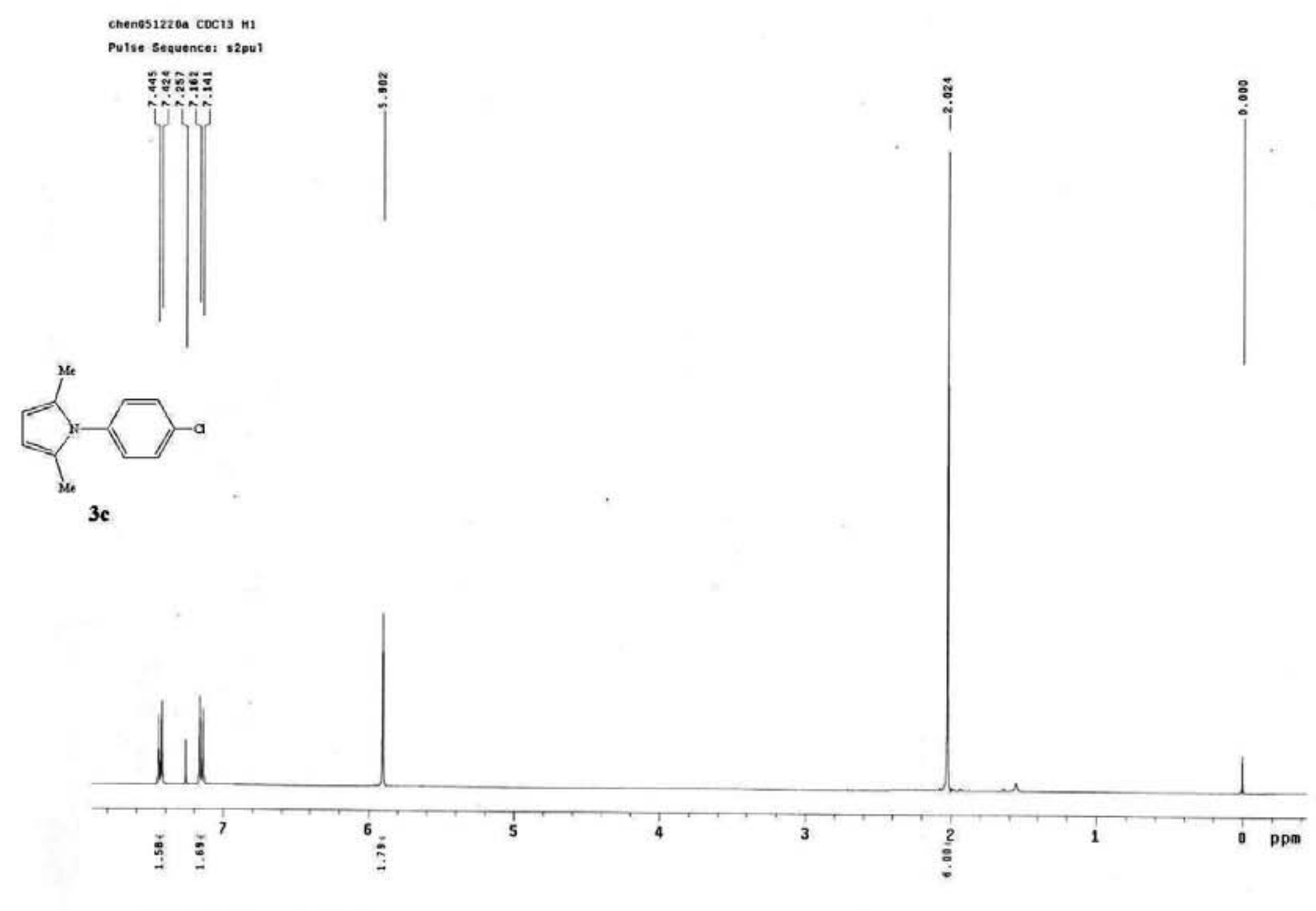

No to Informatrion. use id macko

$$
\begin{aligned}
& \text { enenosiz2oa cocis C13 } \\
& \text { pulse sequencet szpul }
\end{aligned}
$$
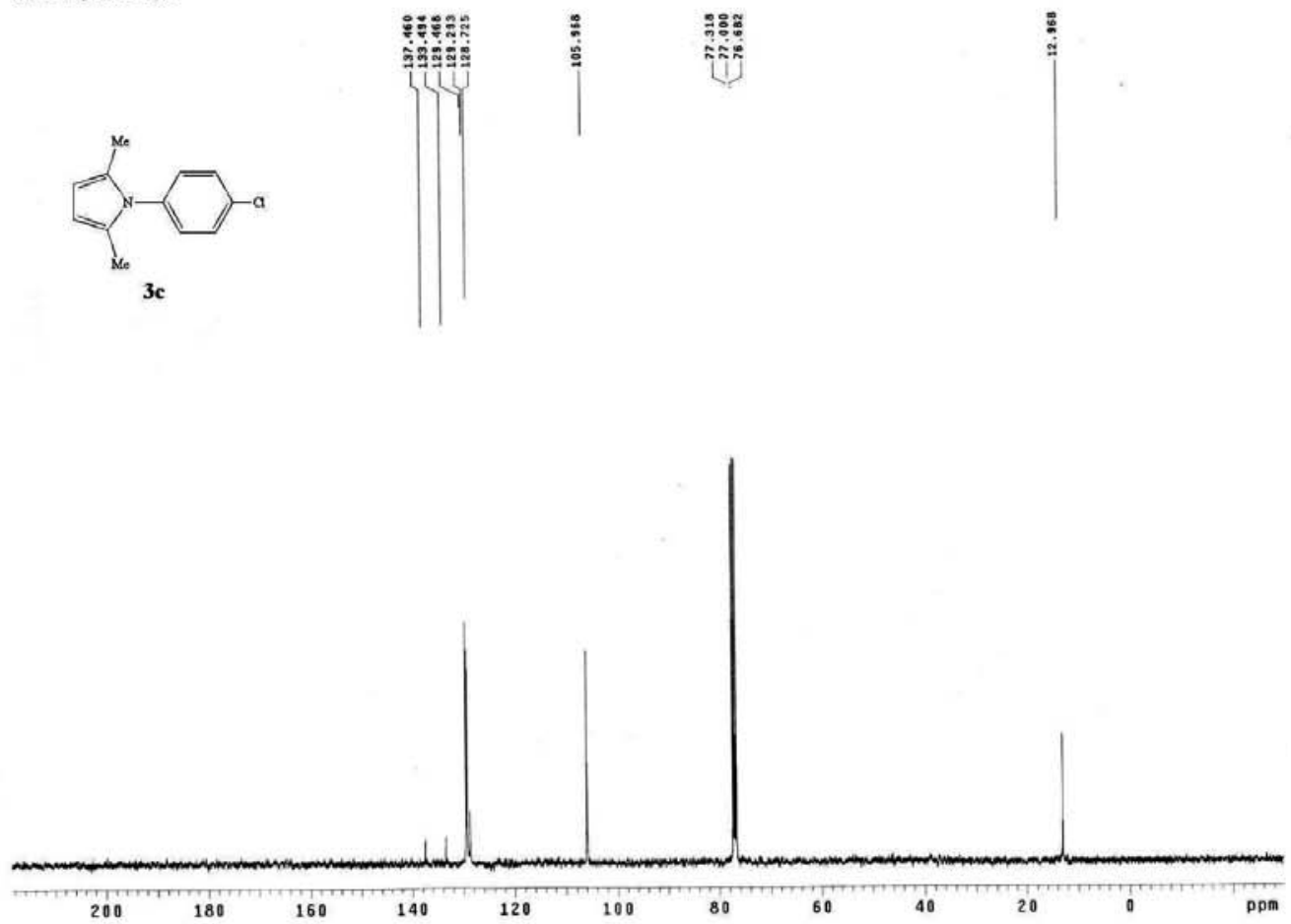

Figure S3. ${ }^{1} \mathrm{H} \mathrm{NMR}$ of $\mathbf{3 c}\left(400 \mathrm{MHz}, \mathrm{CDCl}_{3}\right)$ and ${ }^{13} \mathrm{C} \mathrm{NMR}$ of $\mathbf{3 c}\left(100 \mathrm{MHz}, \mathrm{CDCl}_{3}\right)$. 


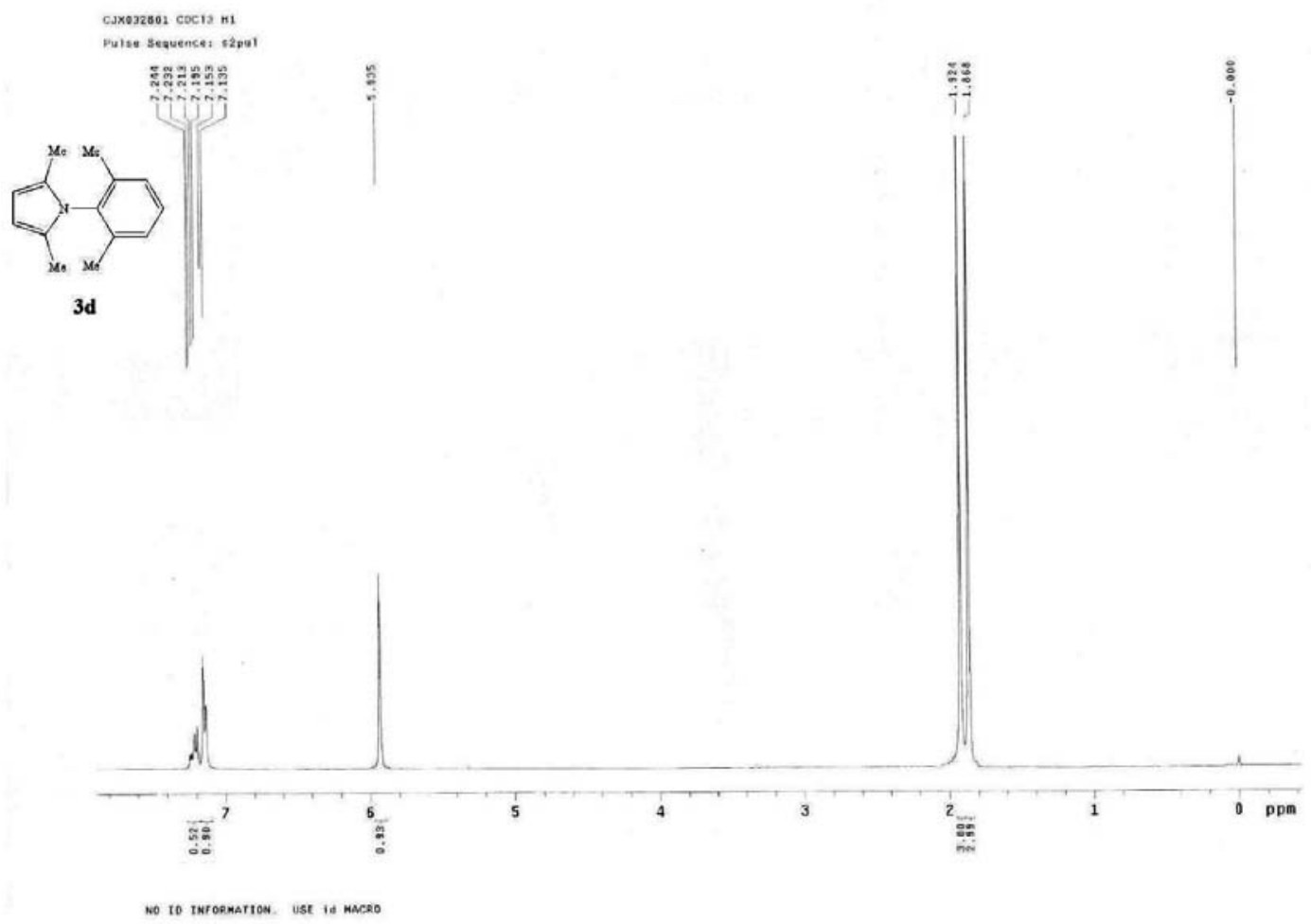

CXNoszon cacis e13

Pulse secuencest s2put

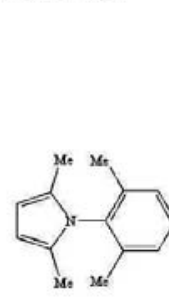

3d

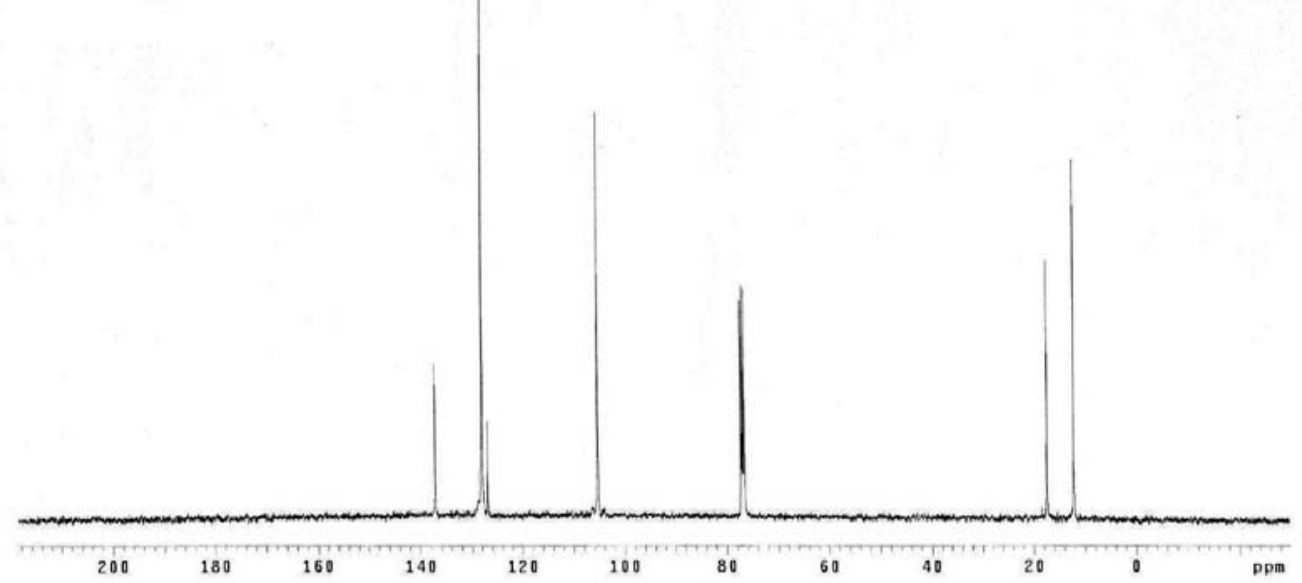

Figure S4. ${ }^{1} \mathrm{H} \mathrm{NMR}$ of $\mathbf{3 d}\left(400 \mathrm{MHz}, \mathrm{CDCl}_{3}\right)$ and ${ }^{13} \mathrm{C} \mathrm{NMR}$ of $\mathbf{3 d}\left(100 \mathrm{MHz}, \mathrm{CDCl}_{3}\right)$. 


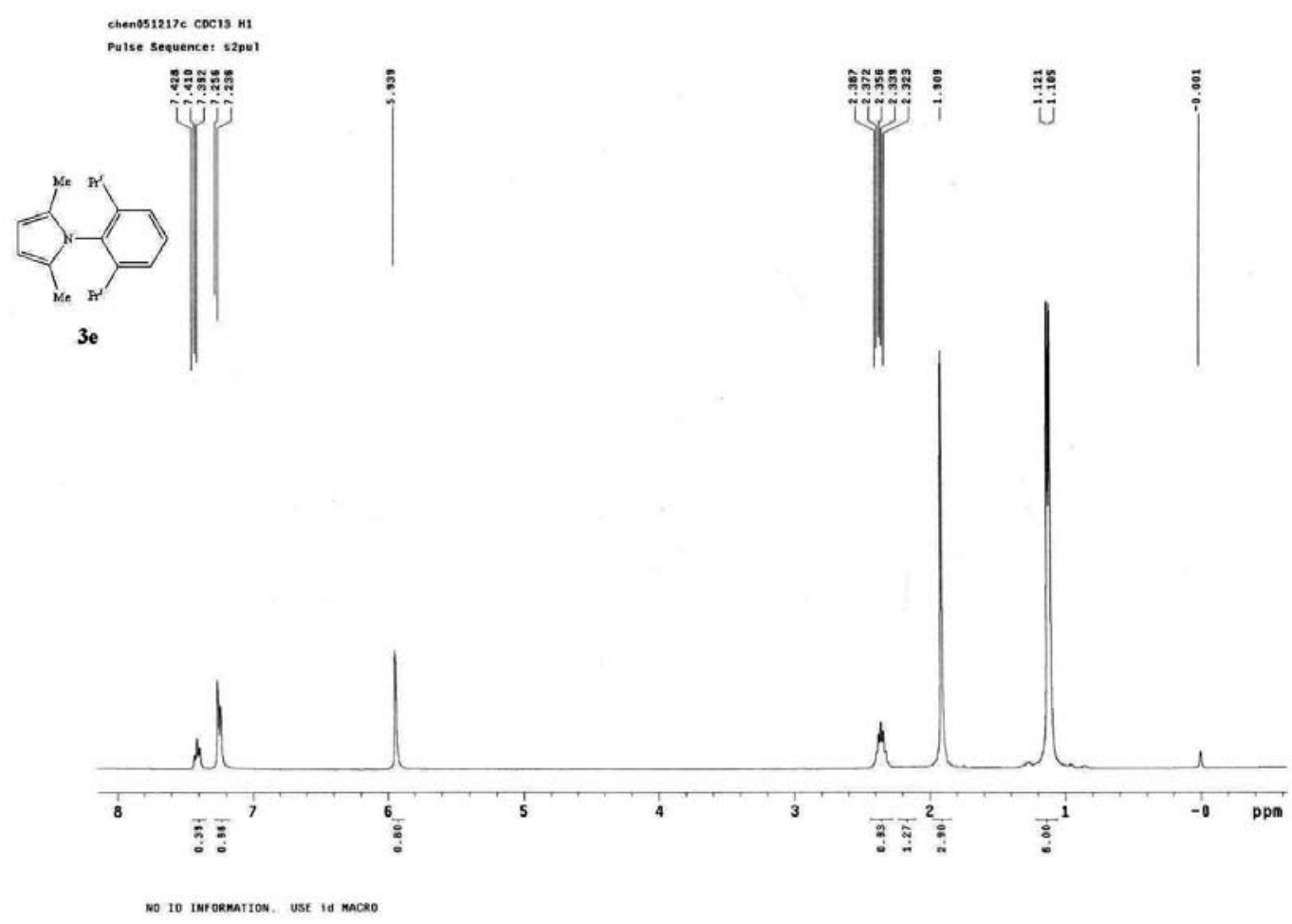

chenosizize cocis C13

pulse Sequences: s2pul
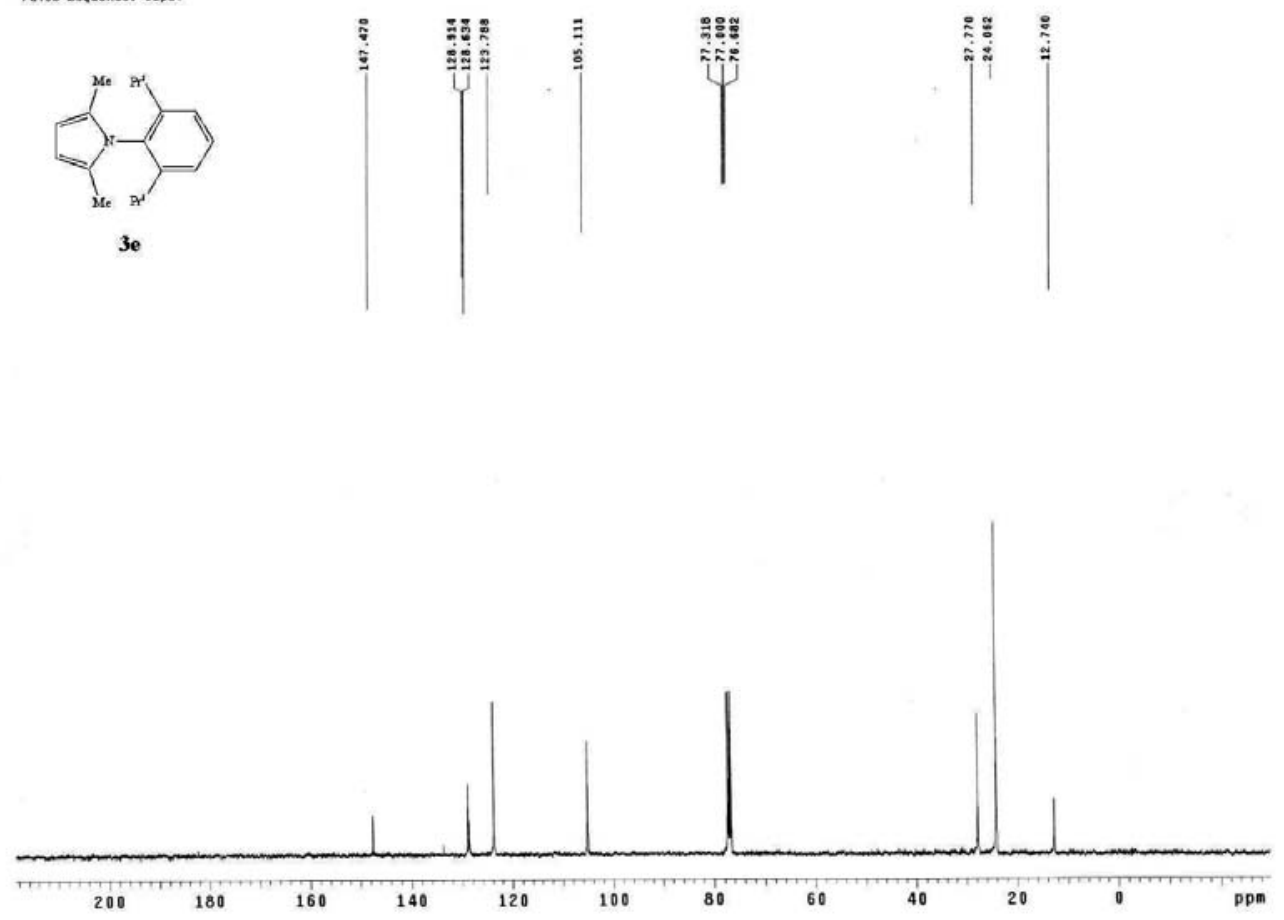

Figure S5. ${ }^{1} \mathrm{H}$ NMR of $\mathbf{3 e}\left(400 \mathrm{MHz}, \mathrm{CDCl}_{3}\right)$ and ${ }^{13} \mathrm{C} \mathrm{NMR}$ of $\mathbf{3 e}\left(100 \mathrm{MHz}, \mathrm{CDCl}_{3}\right)$. 


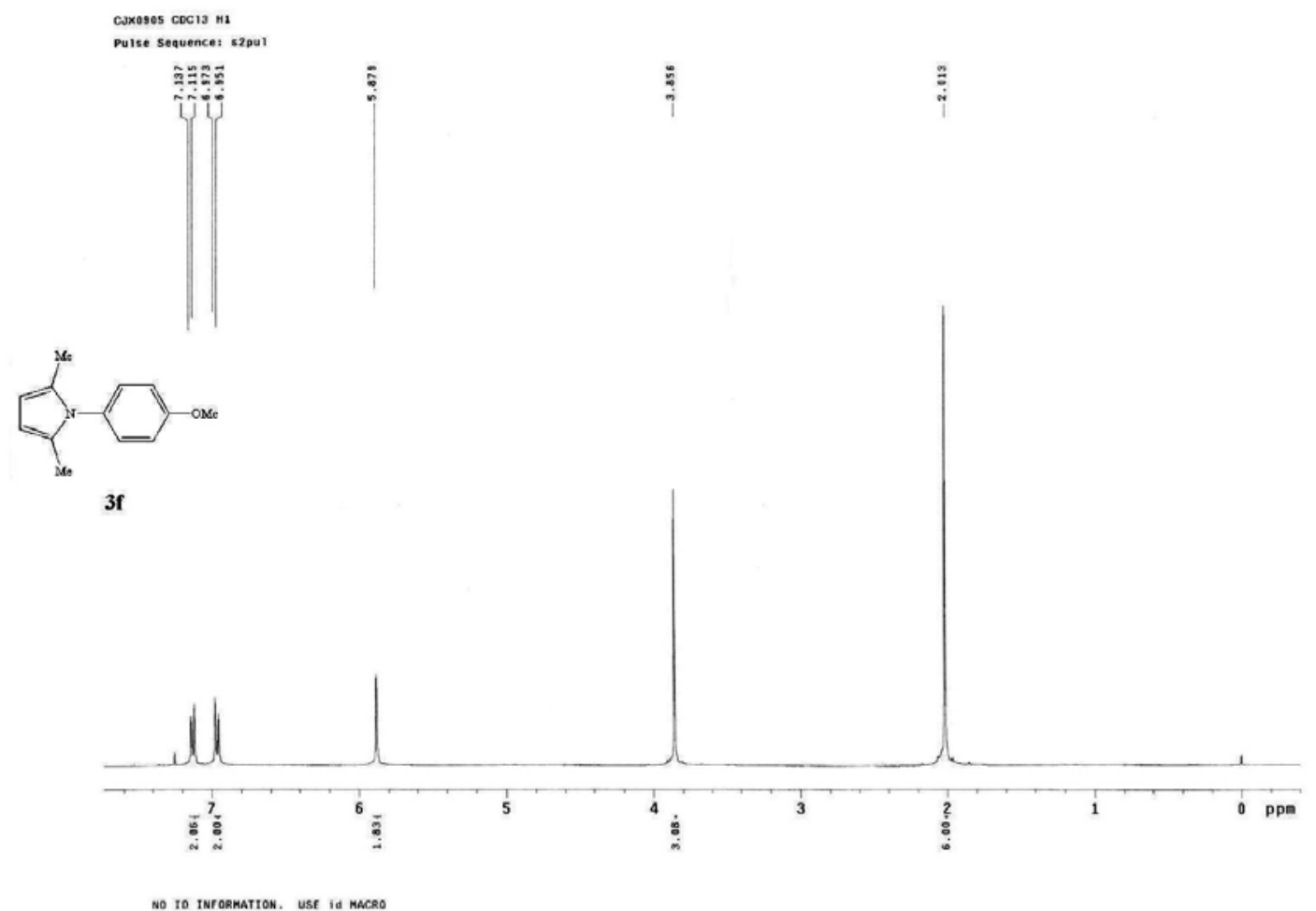

No to twroknatron. USE id Macro

caxosos $\mathrm{CDC}_{13} \mathrm{C} 13$
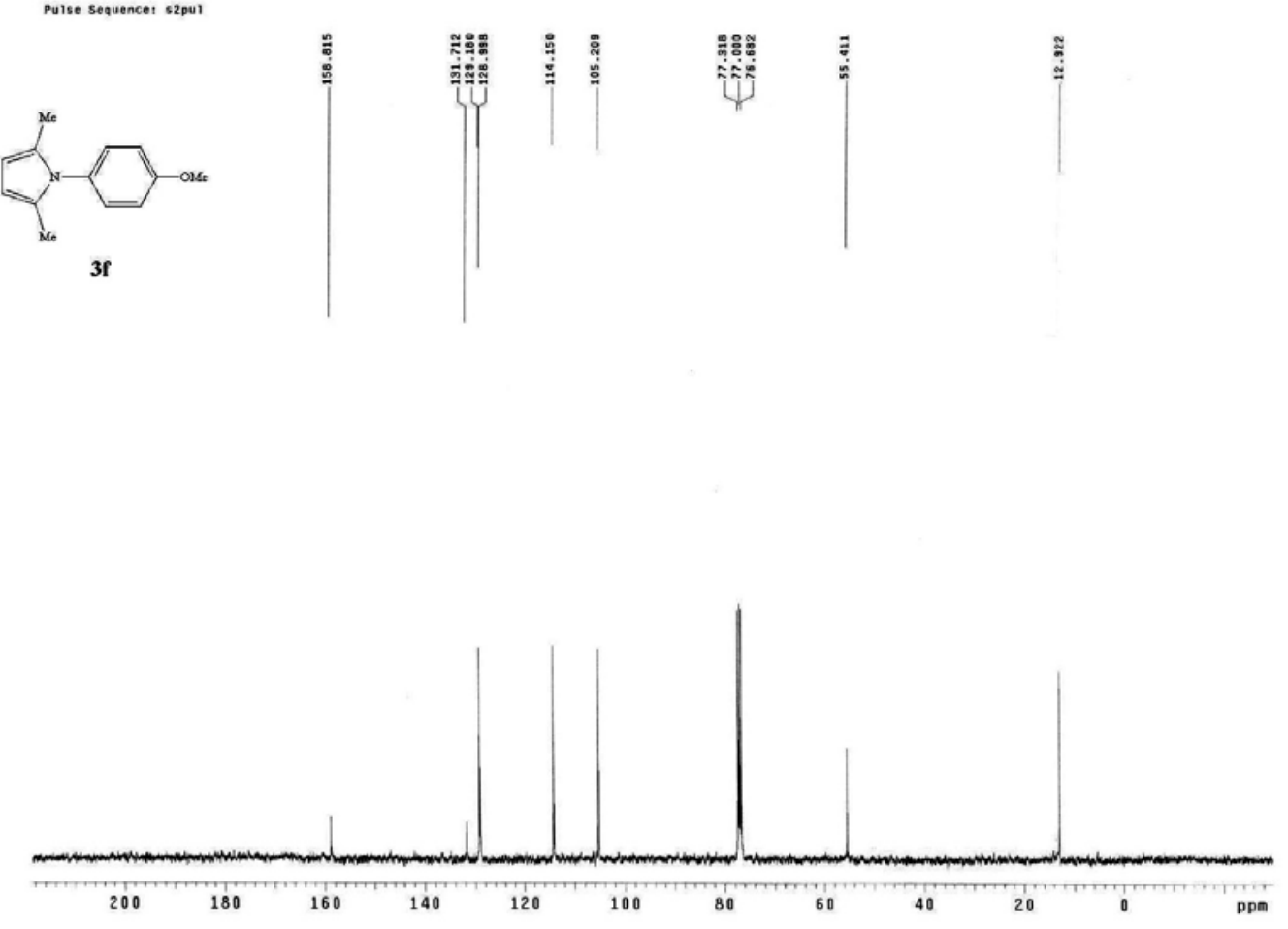

Figure S6. ${ }^{1} \mathrm{H}$ NMR of $\mathbf{3 f}\left(400 \mathrm{MHz}, \mathrm{CDCl}_{3}\right)$ and ${ }^{13} \mathrm{C} \mathrm{NMR}$ of $\mathbf{3 f}\left(100 \mathrm{MHz}, \mathrm{CDCl}_{3}\right)$. 


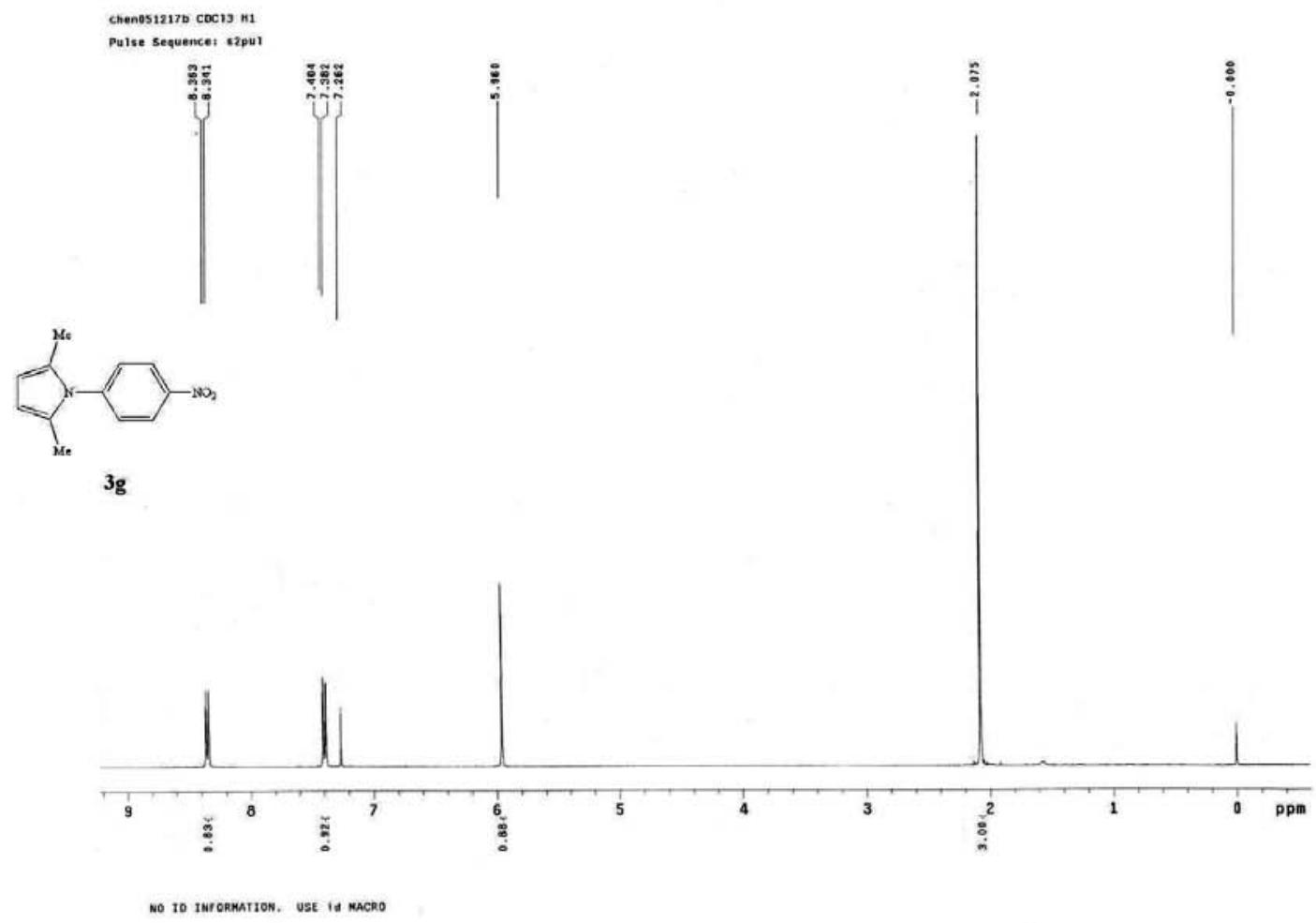

chenes1217b CDC13 C13

Pulse sequences s2pur

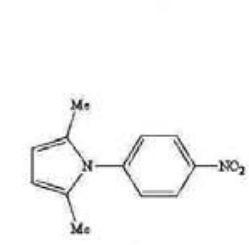

$3 g$
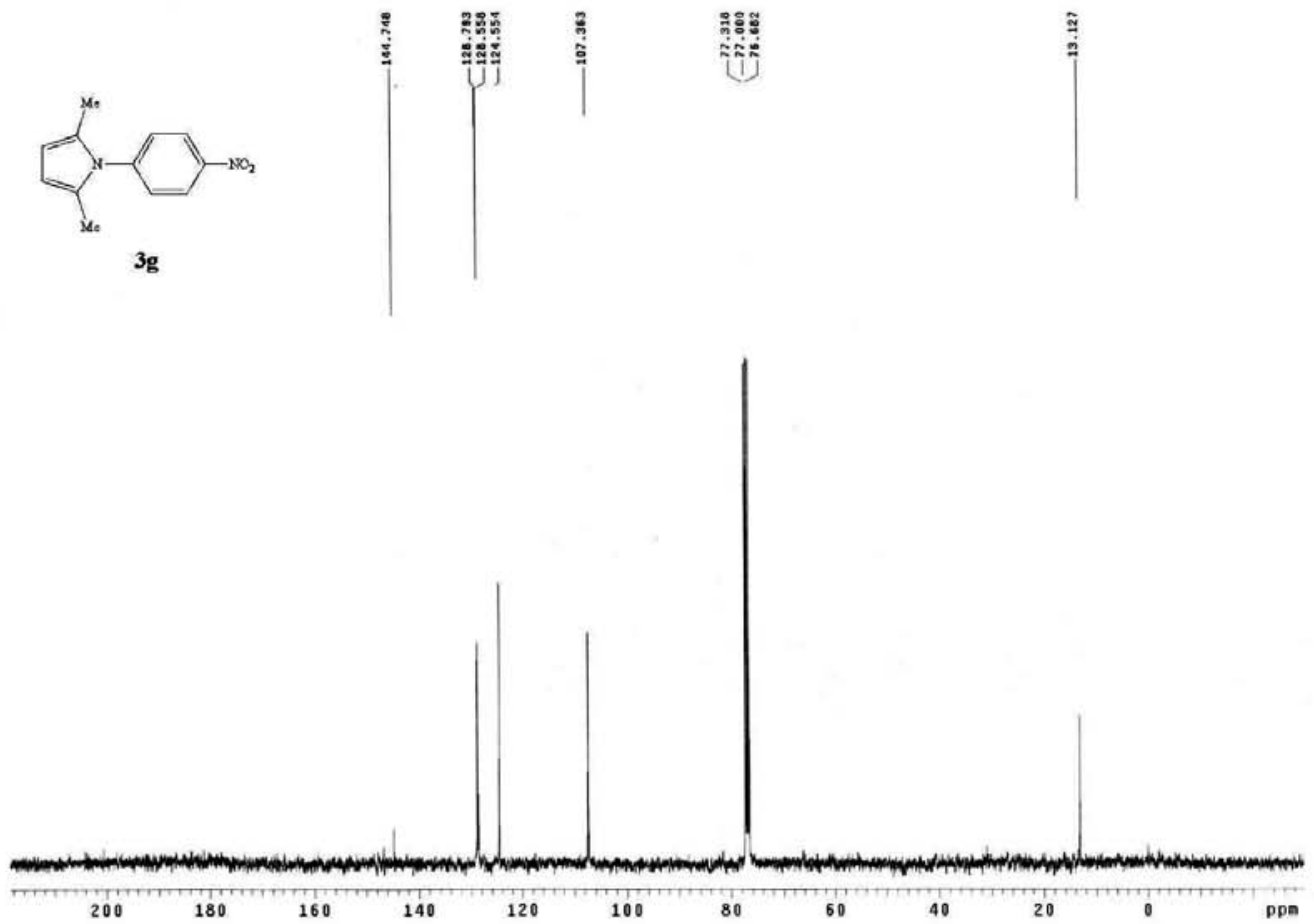

Figure S7. ${ }^{1} \mathrm{H}$ NMR of $\mathbf{3 g}\left(400 \mathrm{MHz} \mathrm{CDCl}_{3}\right)$ and ${ }^{13} \mathrm{C} \mathrm{NMR}$ of $\mathbf{3 g}\left(100 \mathrm{MHz}, \mathrm{CDCl}_{3}\right)$. 

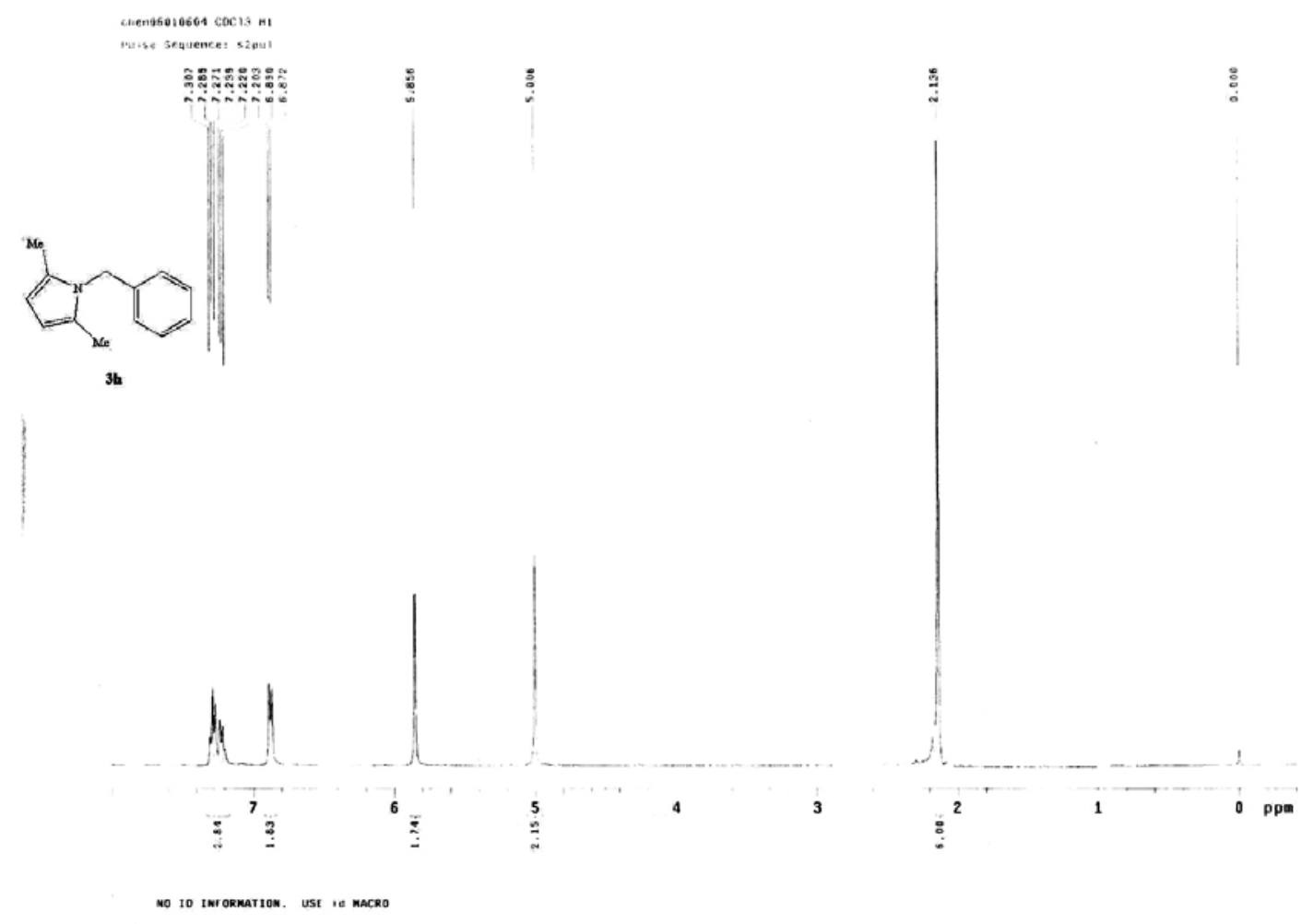

\section{chenesososo4 COC13 C13 \\ Pulse sequencer: s2pur}
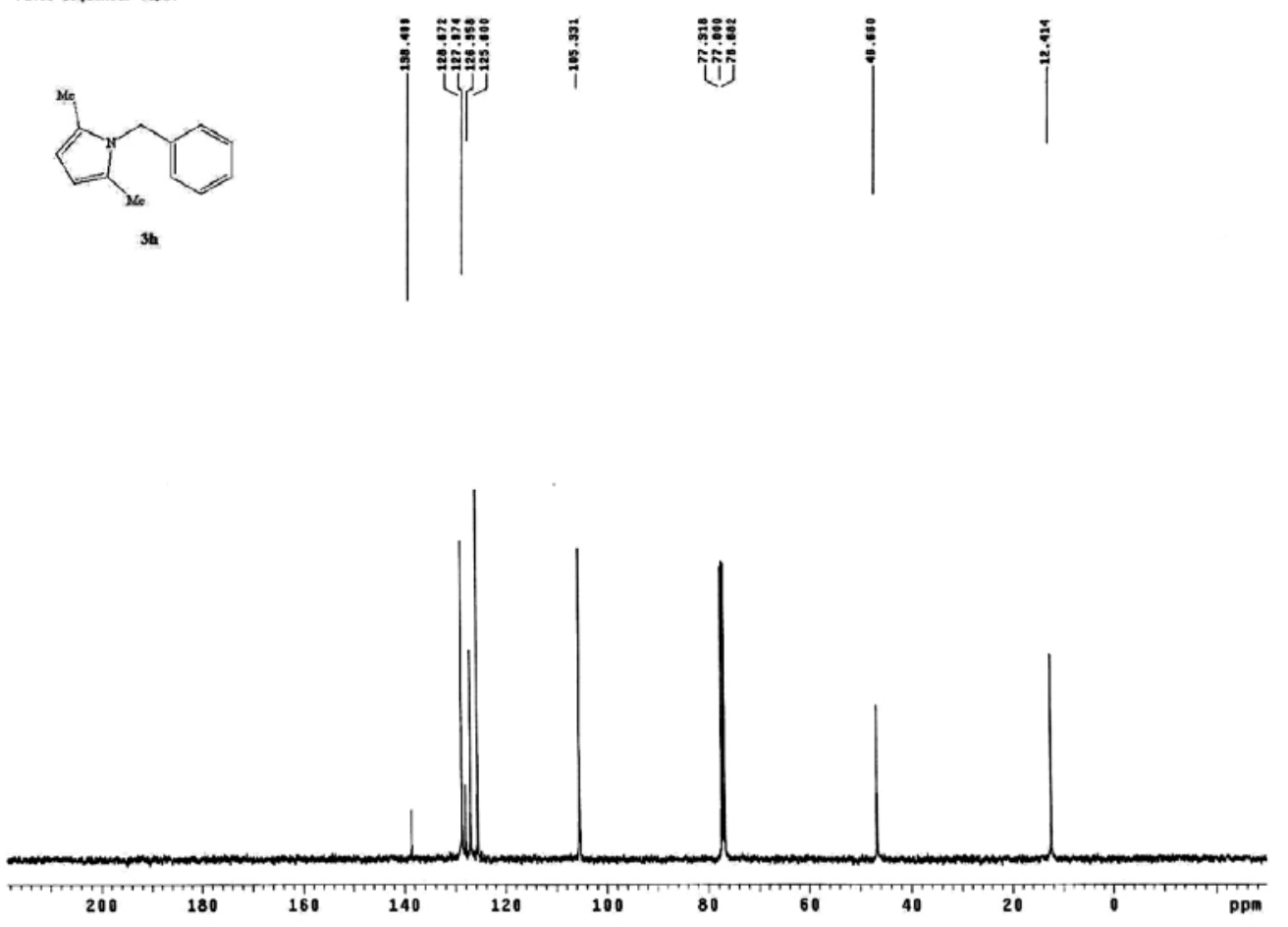

Figure S8. ${ }^{1} \mathrm{H}$ NMR of $\mathbf{3 h}\left(400 \mathrm{MHz}, \mathrm{CDCl}_{3}\right)$ and ${ }^{13} \mathrm{C} \mathrm{NMR}$ of $\mathbf{3 h}\left(100 \mathrm{MHz}, \mathrm{CDCl}_{3}\right)$. 

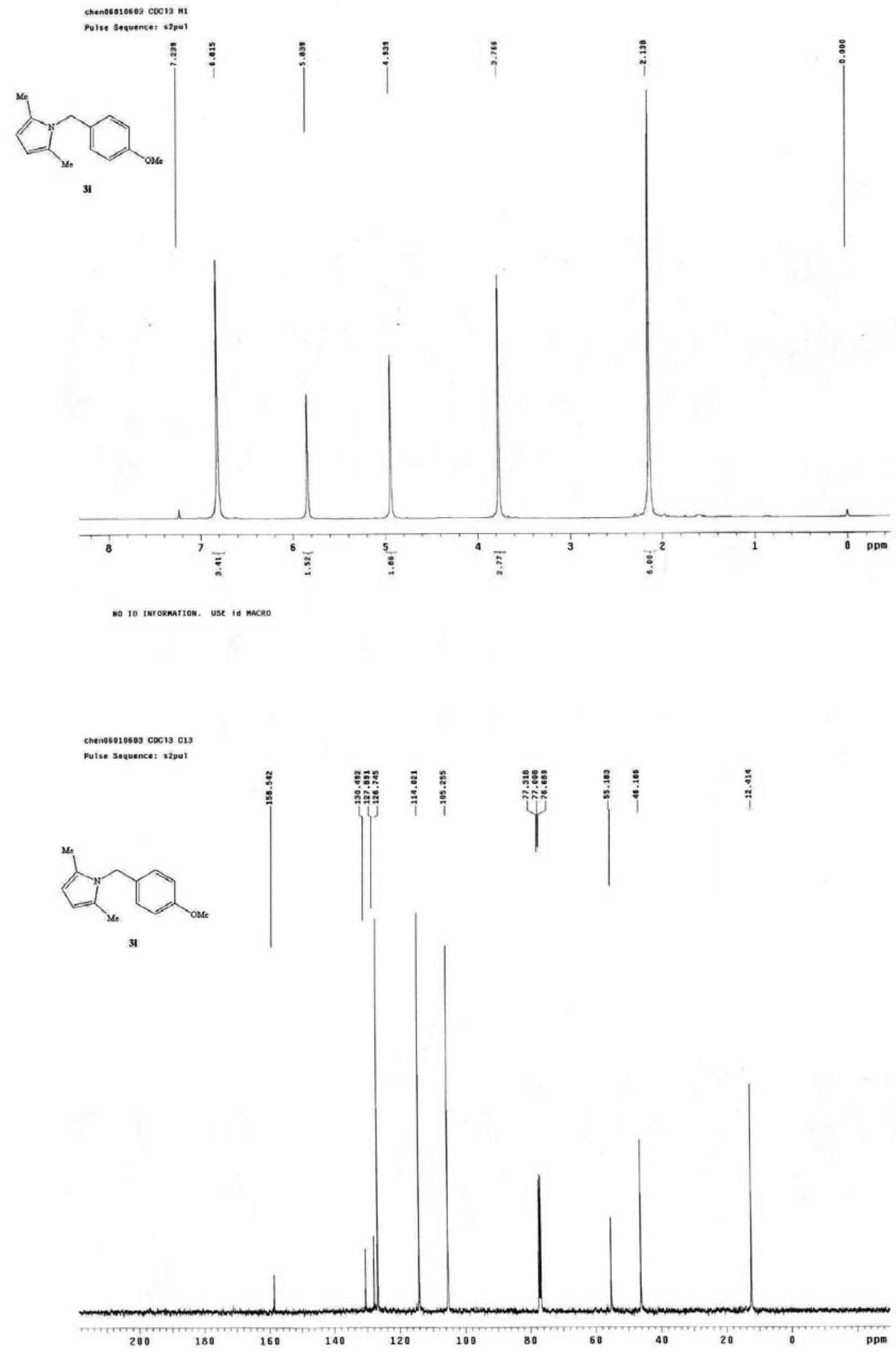

Figure S9. ${ }^{1} \mathrm{H}$ NMR of $\mathbf{3 i}\left(400 \mathrm{MHz}, \mathrm{CDCl}_{3}\right)$ and ${ }^{13} \mathrm{C} \mathrm{NMR}$ of $\mathbf{3 i}\left(100 \mathrm{MHz}, \mathrm{CDCl}_{3}\right)$. 


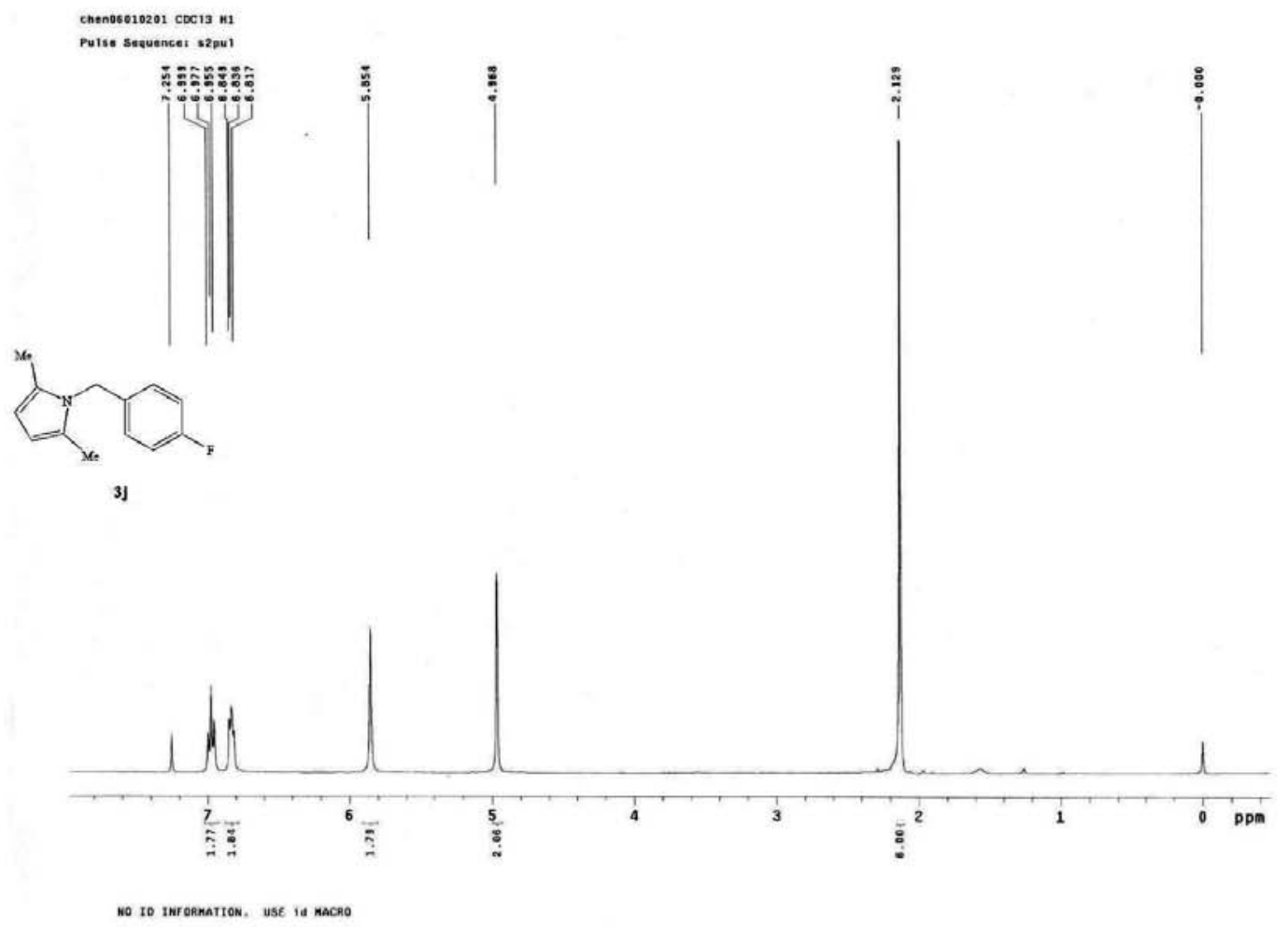

$$
\begin{aligned}
& \text { Cheno6010201 cocis c13 } \\
& \text { Pulse sequenca: e2pul }
\end{aligned}
$$
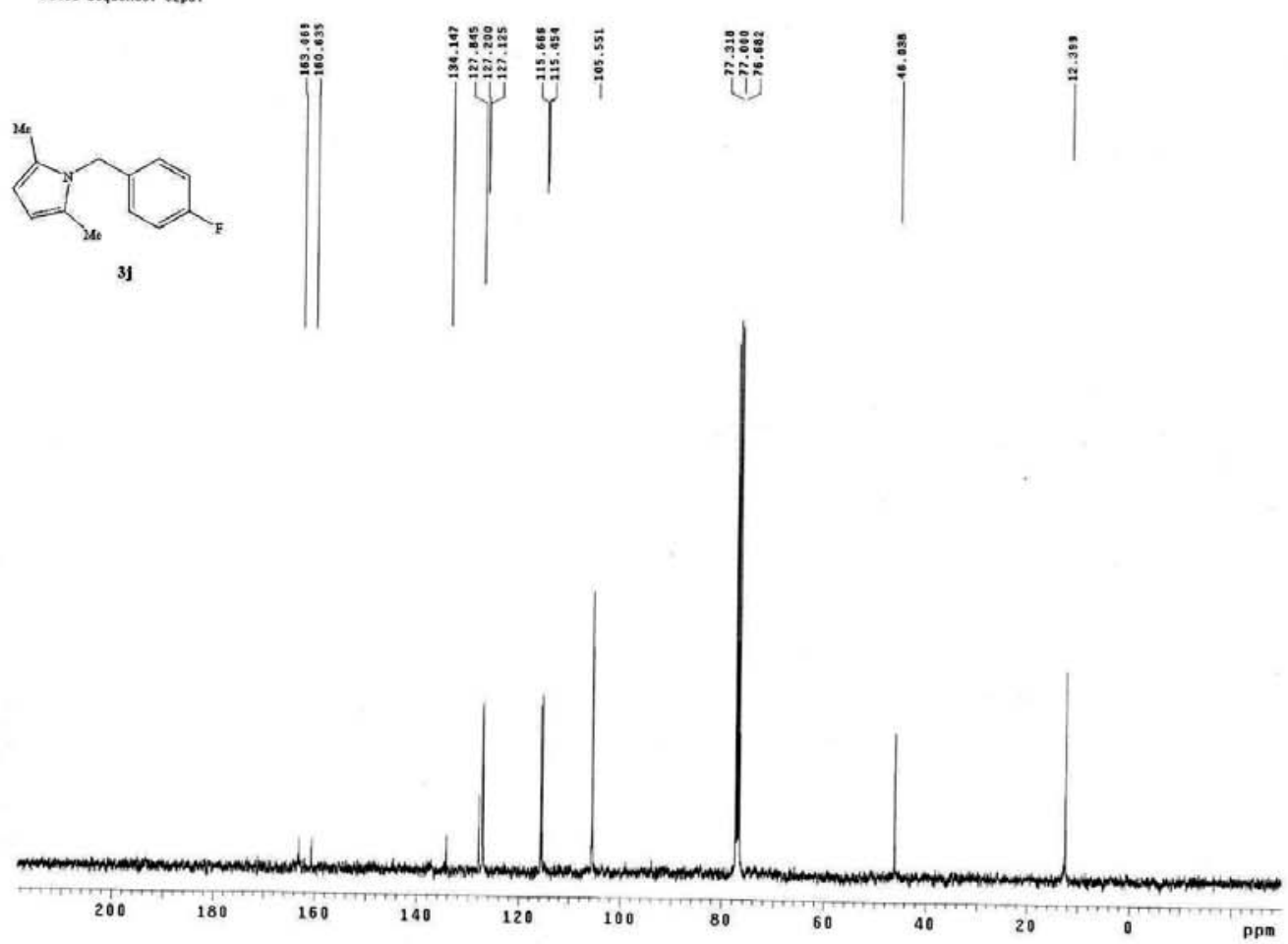

Figure S10. ${ }^{1} \mathrm{H}$ NMR of $\mathbf{3 j}\left(400 \mathrm{MHz}, \mathrm{CDCl}_{3}\right)$ and ${ }^{13} \mathrm{C} \mathrm{NMR}$ of $\mathbf{3 j}\left(100 \mathrm{MHz}, \mathrm{CDCl}_{3}\right)$. 


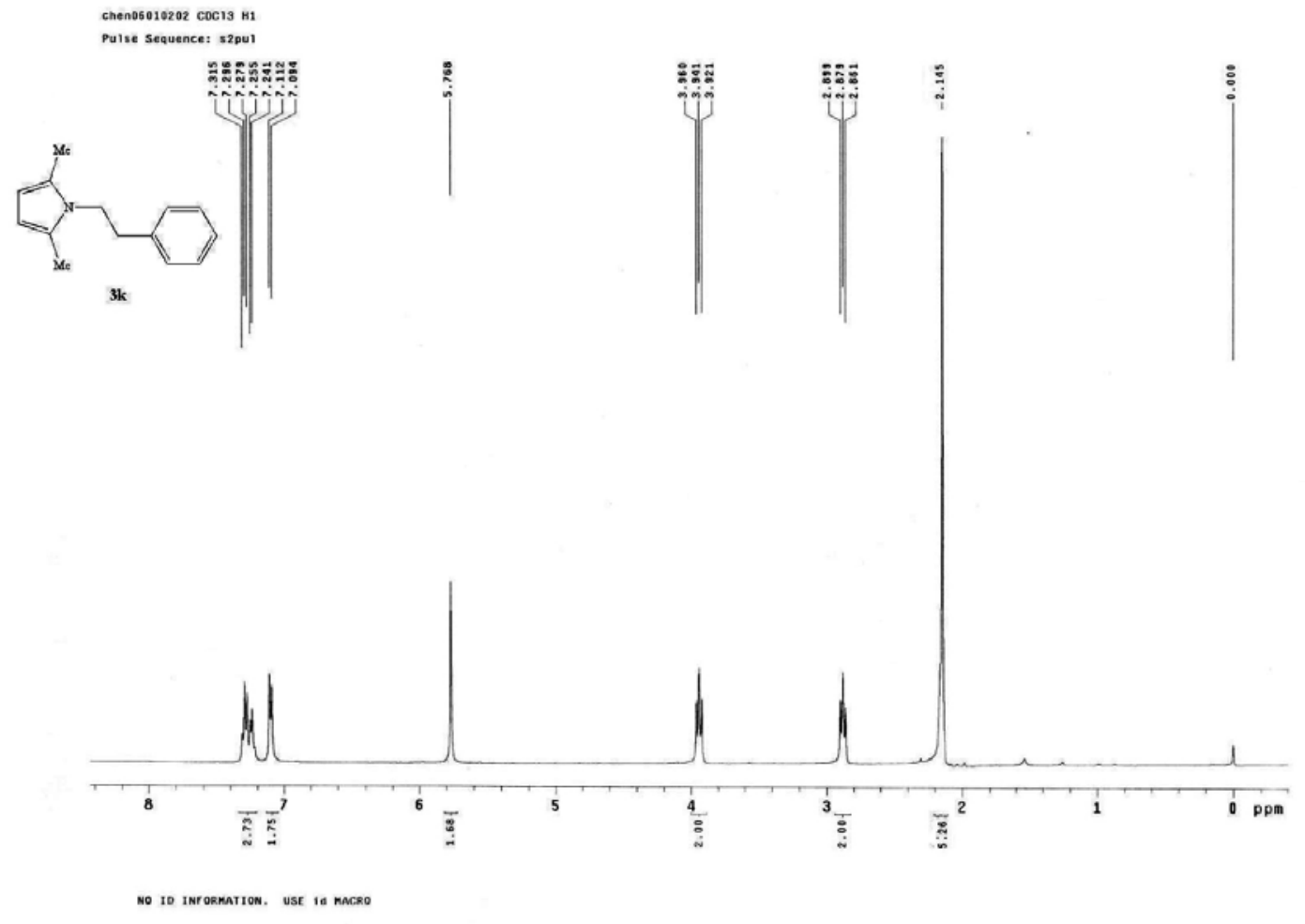

Chen06010202 coc1s C13
Puise sequence: s2pul

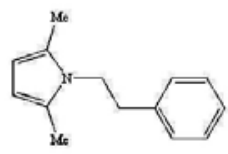

$3 \mathbf{k}$
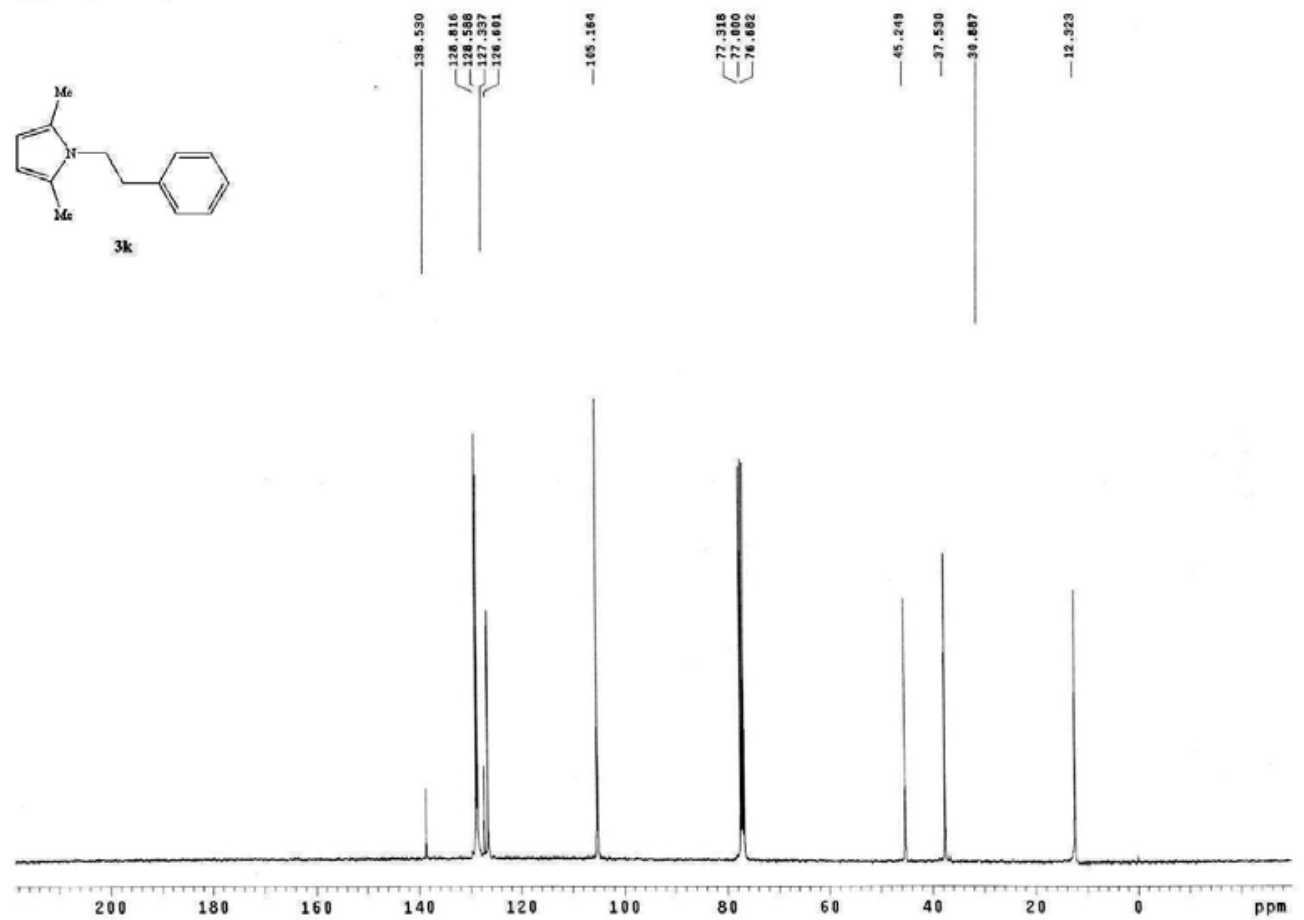

Figure S11. ${ }^{1} \mathrm{H}$ NMR of $\mathbf{3 k}\left(400 \mathrm{MHz}, \mathrm{CDCl}_{3}\right)$ and ${ }^{13} \mathrm{C} \mathrm{NMR}$ of $\mathbf{3 k}\left(100 \mathrm{MHz}, \mathrm{CDCl}_{3}\right)$. 

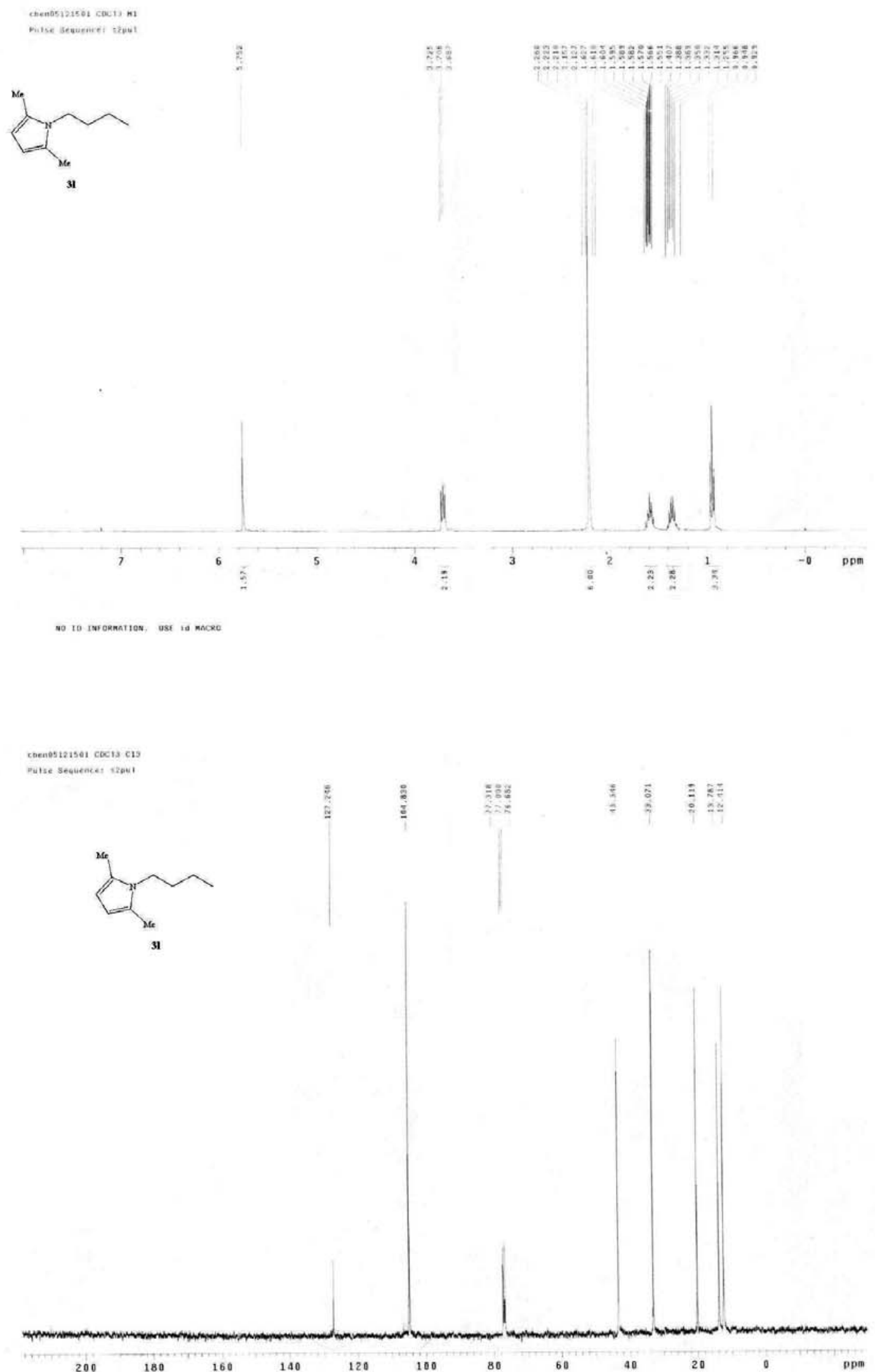

Figure S12. ${ }^{1} \mathrm{H}$ NMR of $\mathbf{3 l}\left(400 \mathrm{MHz}, \mathrm{CDCl}_{3}\right)$ and ${ }^{13} \mathrm{C} \mathrm{NMR}$ of $\mathbf{3 l}\left(100 \mathrm{MHz}, \mathrm{CDCl}_{3}\right)$. 


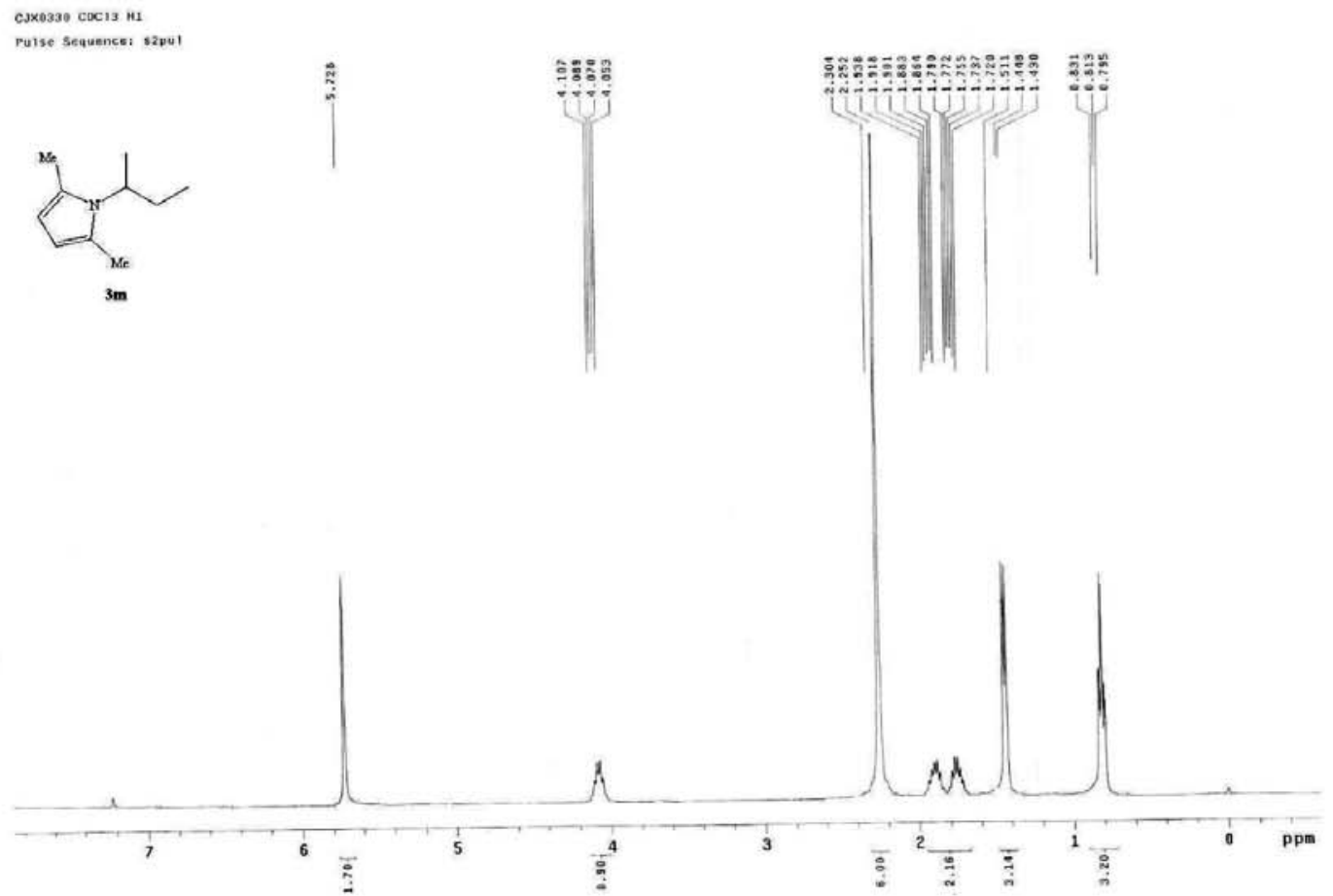

no 10 throRmatron. Use id macro

CJX0300 COC13 C13

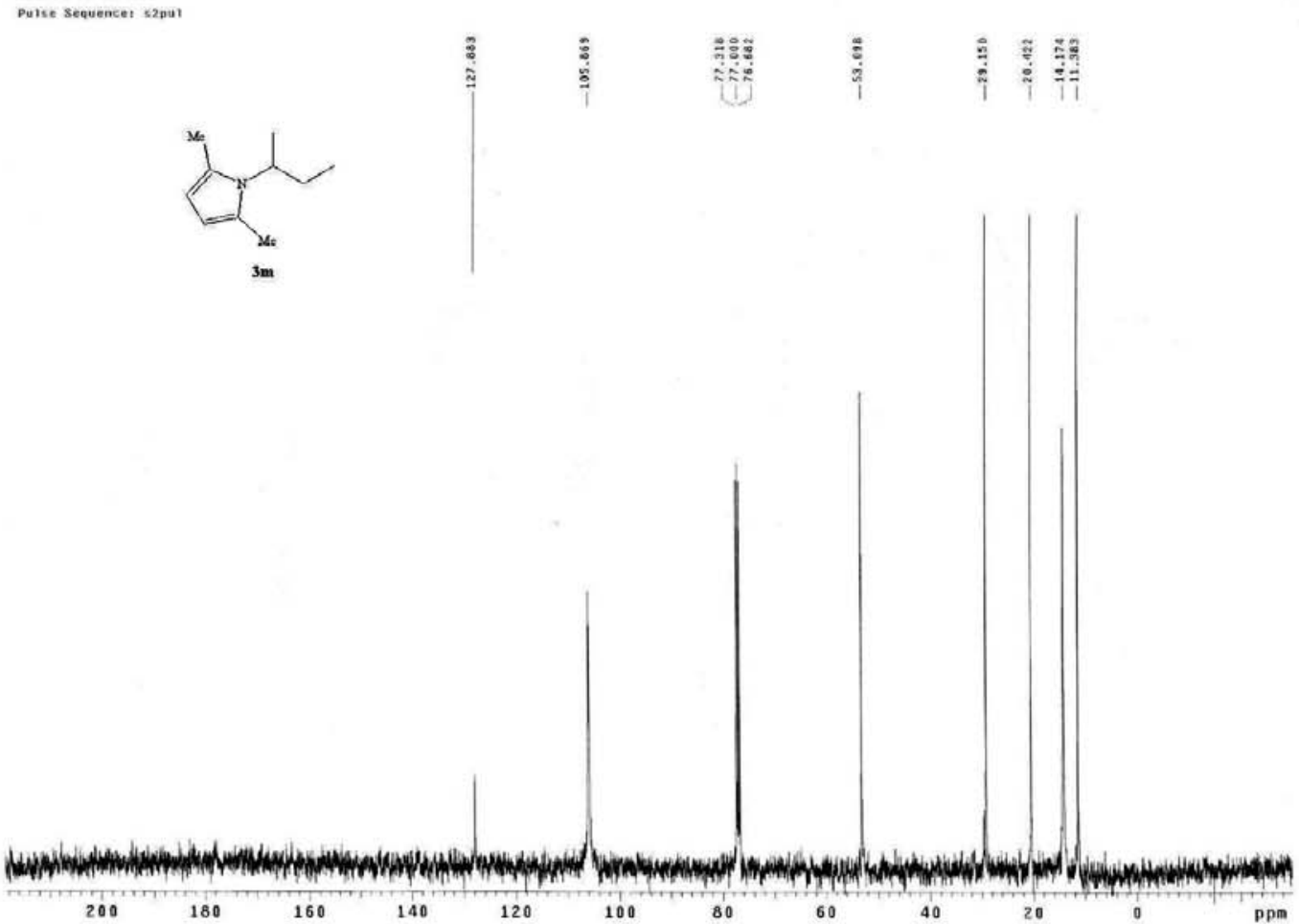

Figure S13. ${ }^{1} \mathrm{H}$ NMR of $\mathbf{3 m}\left(400 \mathrm{MHz} \mathrm{CDCl}_{3}\right)$ and ${ }^{13} \mathrm{C} \mathrm{NMR}$ of $\mathbf{3 m}\left(100 \mathrm{MHz}, \mathrm{CDCl}_{3}\right)$. 


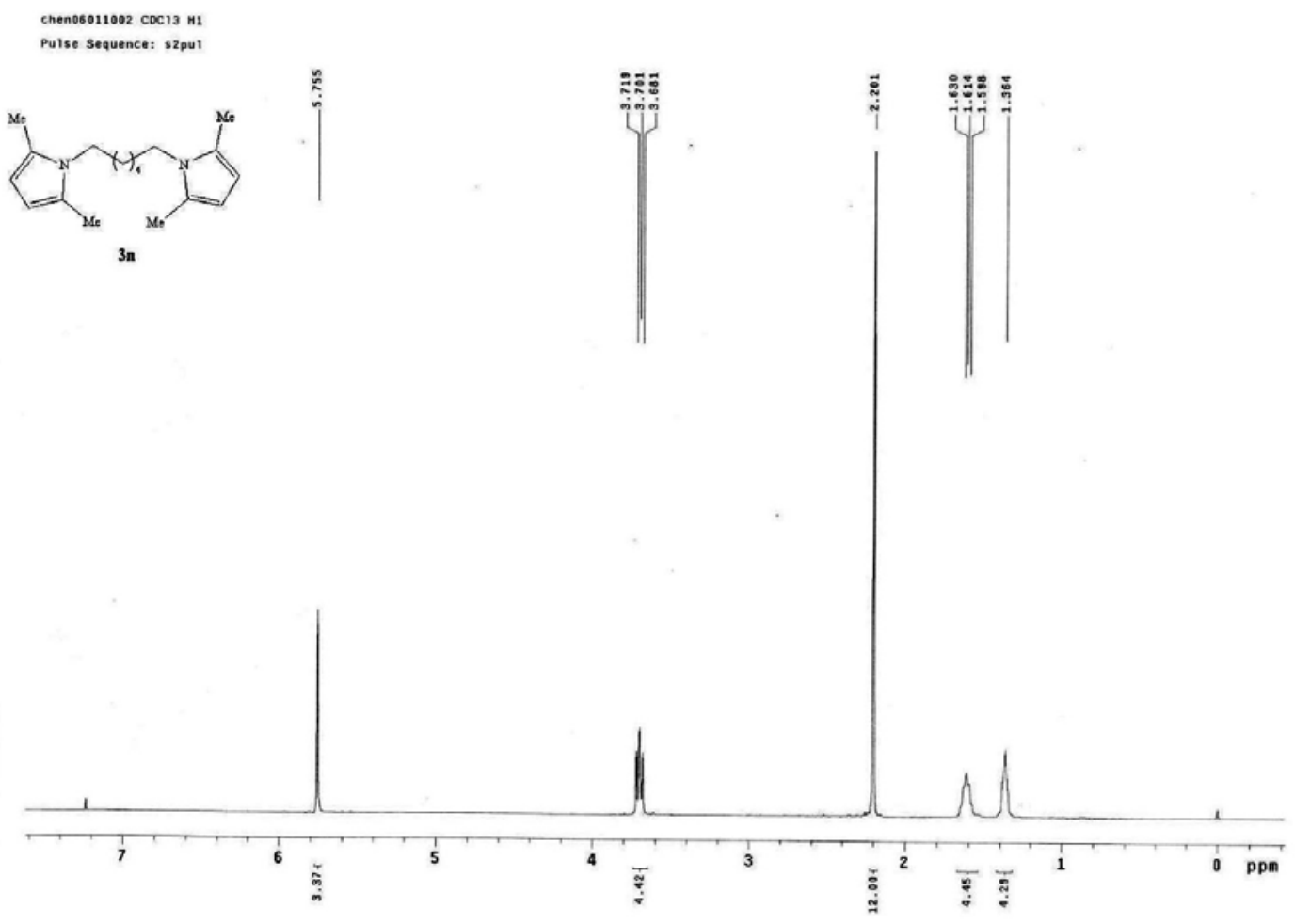

no to Informattow. use ia macro

\section{Chen06011002 COC13 C13}

Pulse sequence: s2pur
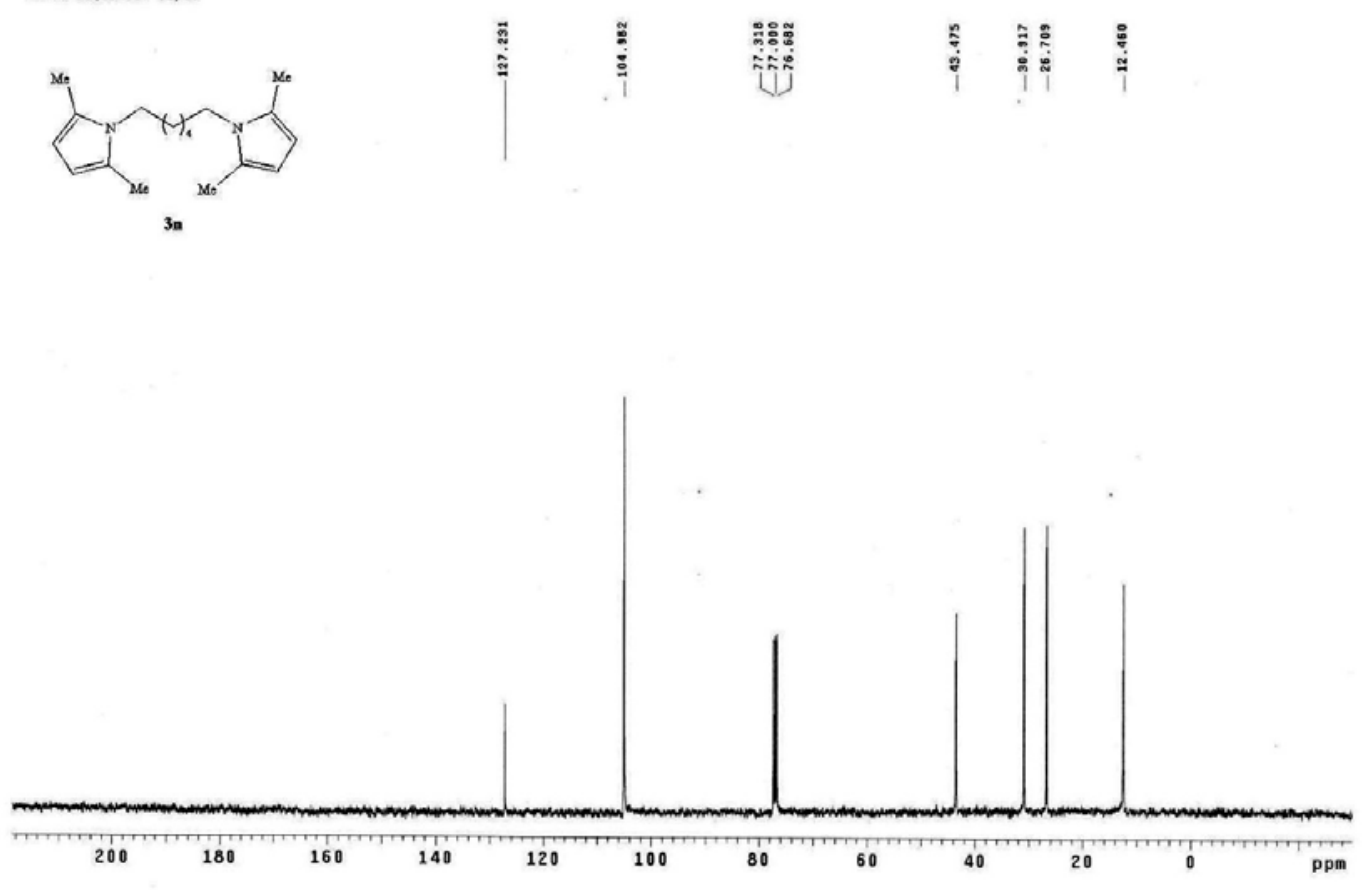

Figure S14. ${ }^{1} \mathrm{H} \mathrm{NMR}$ of $\mathbf{3 n}\left(400 \mathrm{MHz}, \mathrm{CDCl}_{3}\right)$ and ${ }^{13} \mathrm{C} \mathrm{NMR}$ of $\mathbf{3 n}\left(100 \mathrm{MHz}, \mathrm{CDCl}_{3}\right)$. 


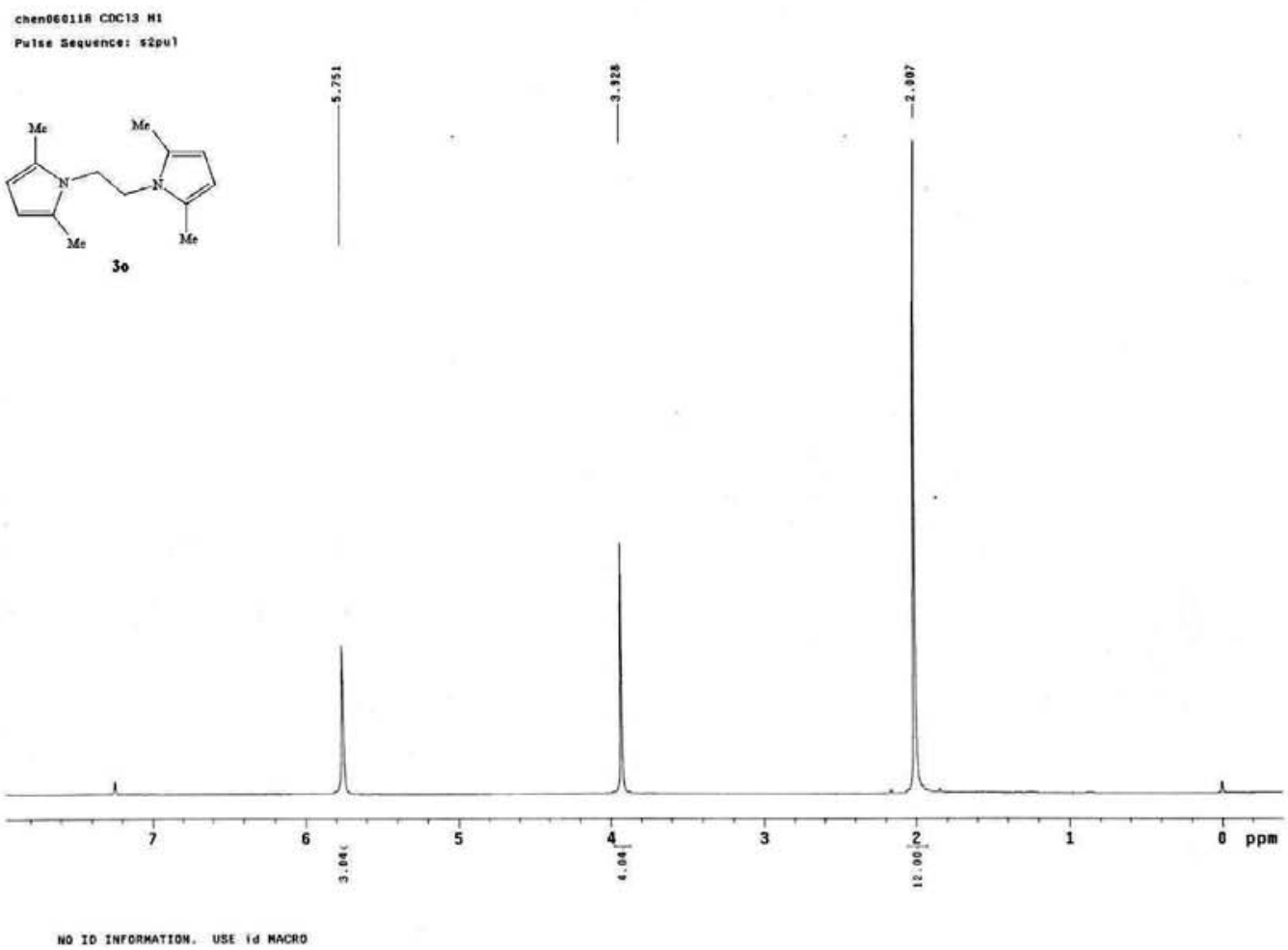

Chen0118 CDC13 C13

Pulse sequences s2put
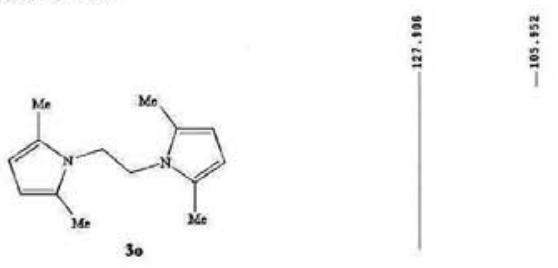

कิ
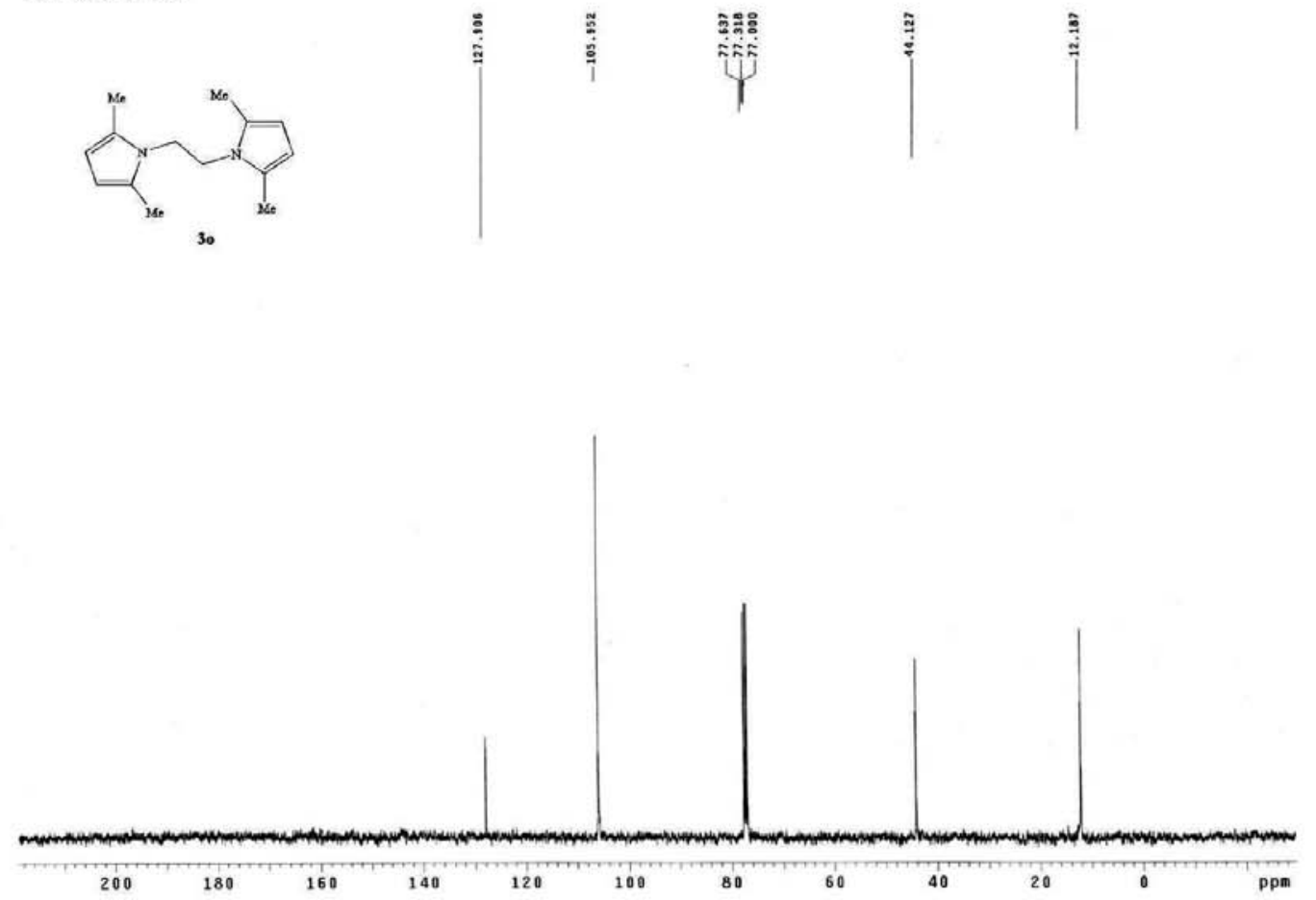

Figure S15. ${ }^{1} \mathrm{H}$ NMR of $3 \mathbf{o}\left(400 \mathrm{MHz}, \mathrm{CDCl}_{3}\right)$ and ${ }^{13} \mathrm{C} \mathrm{NMR}$ of $3 \mathbf{o}\left(100 \mathrm{MHz}, \mathrm{CDCl}_{3}\right)$. 
chenesitizo CDC13 $\mathrm{mz}$

bulse sequancet $32 \mathrm{pi}$
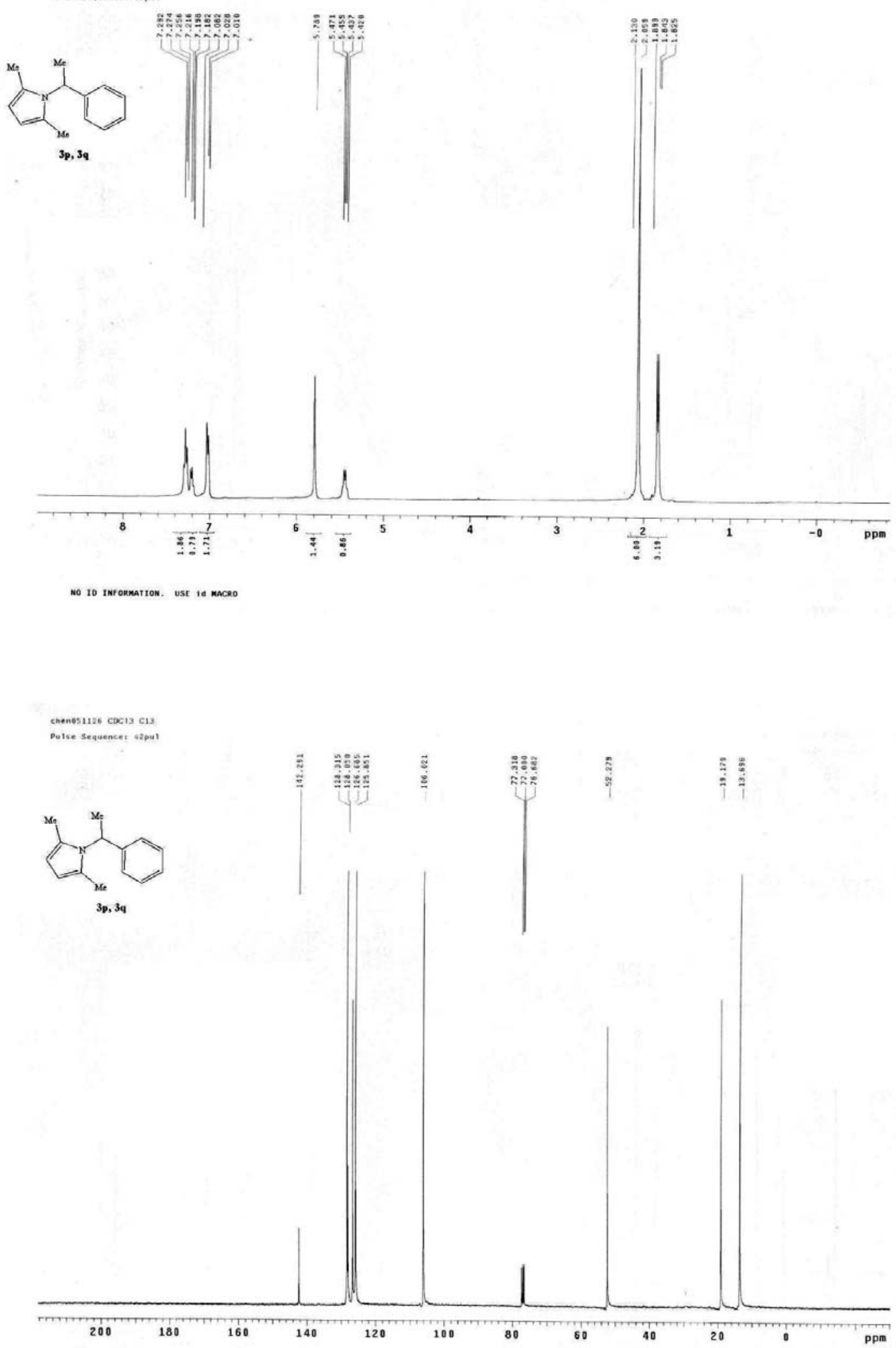

Figure S16. ${ }^{1} \mathrm{H}$ NMR of $3 \mathbf{p}$ or $\mathbf{3 q}\left(400 \mathrm{MHz}, \mathrm{CDCl}_{3}\right)$ and ${ }^{13} \mathrm{C} \mathrm{NMR}$ of $\mathbf{3 p}$ or $\mathbf{3 q}\left(100 \mathrm{MHz}, \mathrm{CDCl}_{3}\right)$. 


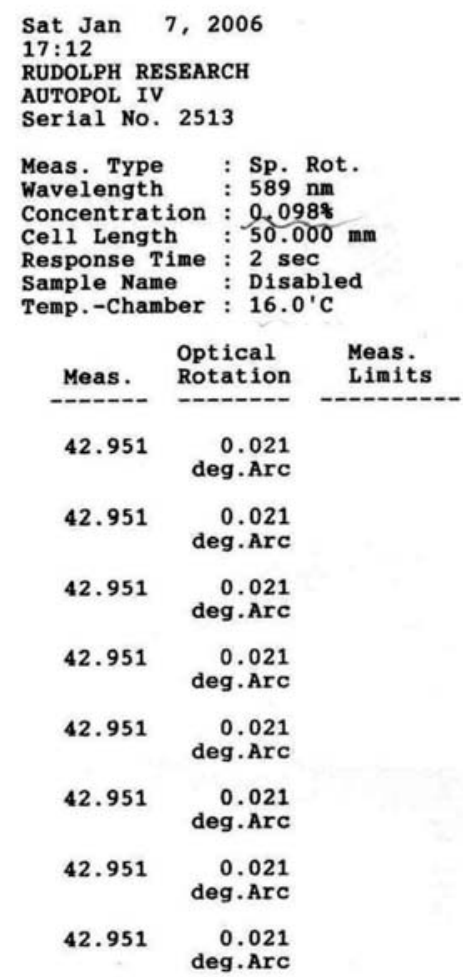

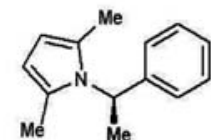

$3 p$

Figure S17. Optical Rotation of 3p.

\begin{tabular}{|c|c|c|}
\hline \multicolumn{3}{|c|}{$\begin{array}{lll}\text { Meas. Type } & \text { Sp. Rot. } \\
\text { Waveiength } & 589 \mathrm{~nm} \\
\text { Concentration } & 0.098 \% \\
\text { Cell Length } & 50.000 \mathrm{~mm} \\
\text { Response Time } & 2 \mathrm{sec} \\
\text { Sample Name } & \text { Disabled } \\
\text { Temp.-Chamber } & 16.9^{\prime} \mathrm{C}\end{array}$} \\
\hline Meas. & $\begin{array}{l}\text { Optical } \\
\text { Rotation }\end{array}$ & $\begin{array}{l}\text { Meas. } \\
\text { Limits }\end{array}$ \\
\hline-42.955 & $\begin{array}{r}-0.021 \\
\text { deg.Arc }\end{array}$ & \\
\hline-42.955 & $\begin{array}{r}-0.021 \\
\text { deg.Arc }\end{array}$ & \\
\hline-42.955 & $\begin{array}{r}-0.021 \\
\text { deg.Arc }\end{array}$ & \\
\hline-42.955 & $\begin{array}{r}-0.021 \\
\text { deg.Arc }\end{array}$ & \\
\hline-42.955 & $\begin{array}{r}-0.021 \\
\text { deg.Arc }\end{array}$ & \\
\hline-42.955 & $\begin{array}{r}-0.021 \\
\text { deg.Arc }\end{array}$ & \\
\hline-42.955 & $\begin{array}{r}-0.021 \\
\text { deg.Arc }\end{array}$ & \\
\hline-42.955 & $\begin{array}{r}-0.021 \\
\text { deg.Arc }\end{array}$ & \\
\hline
\end{tabular}

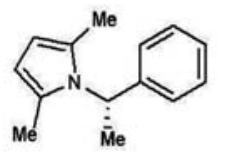

$3 q$

Figure S18. Optical Rotation of 3q. 


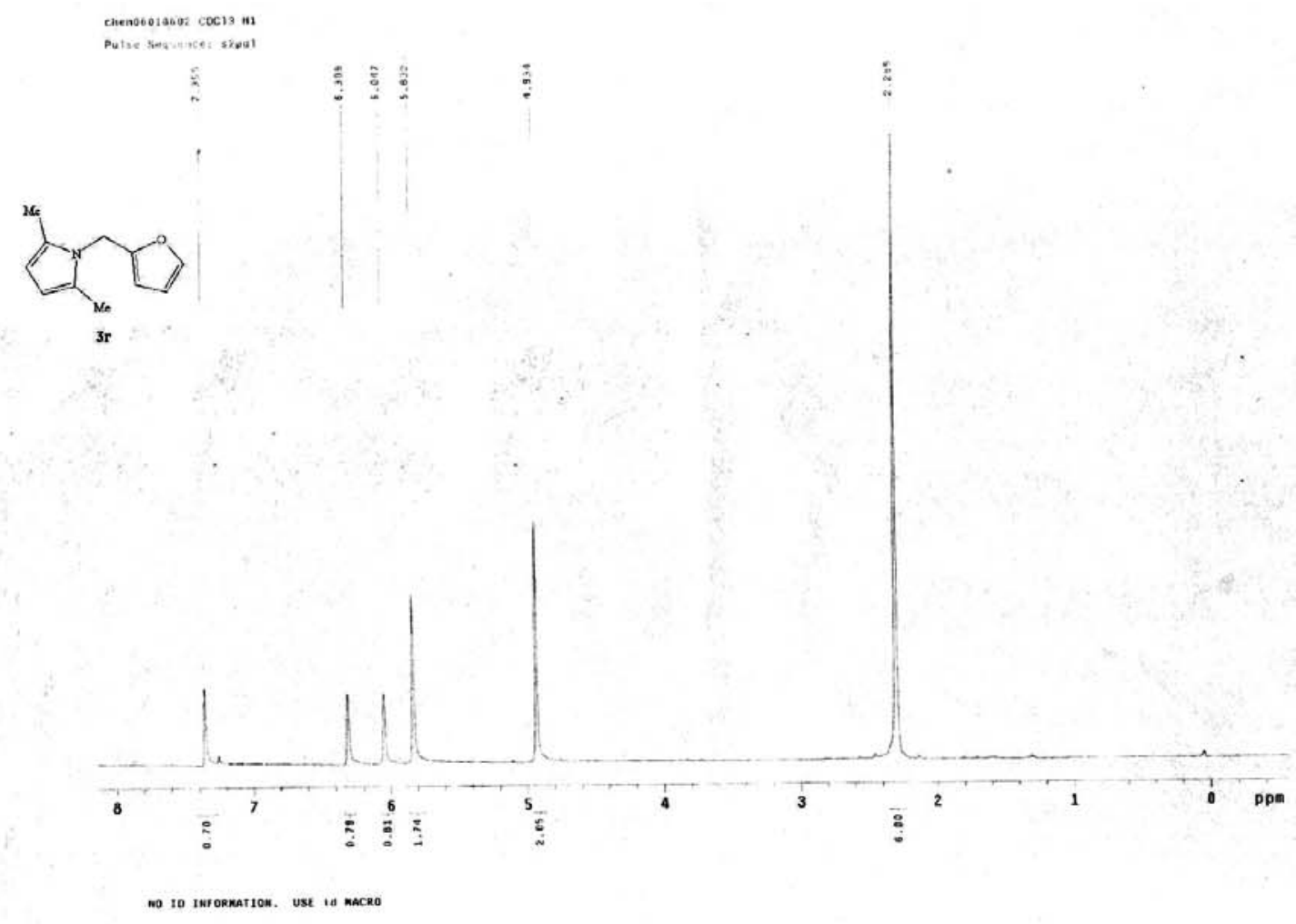

\section{Chenosoroser cocis c1s} Pulse Beguence: s2pul
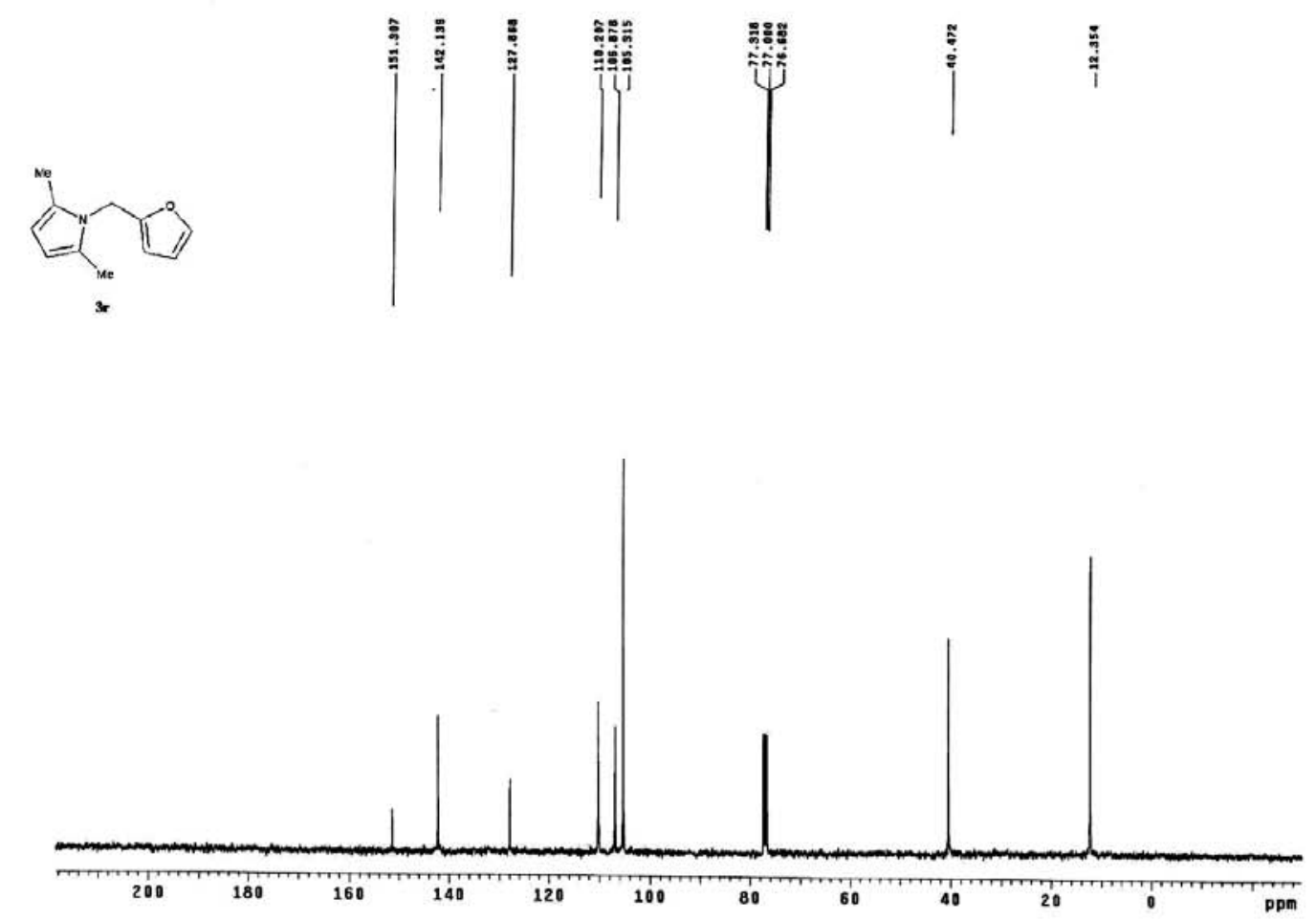

Figure S19. ${ }^{1} \mathrm{H}$ NMR of $\mathbf{3 r}\left(400 \mathrm{MHz}, \mathrm{CDCl}_{3}\right)$ and ${ }^{13} \mathrm{C} \mathrm{NMR}$ of $\mathbf{3 r}\left(100 \mathrm{MHz}, \mathrm{CDCl}_{3}\right)$. 


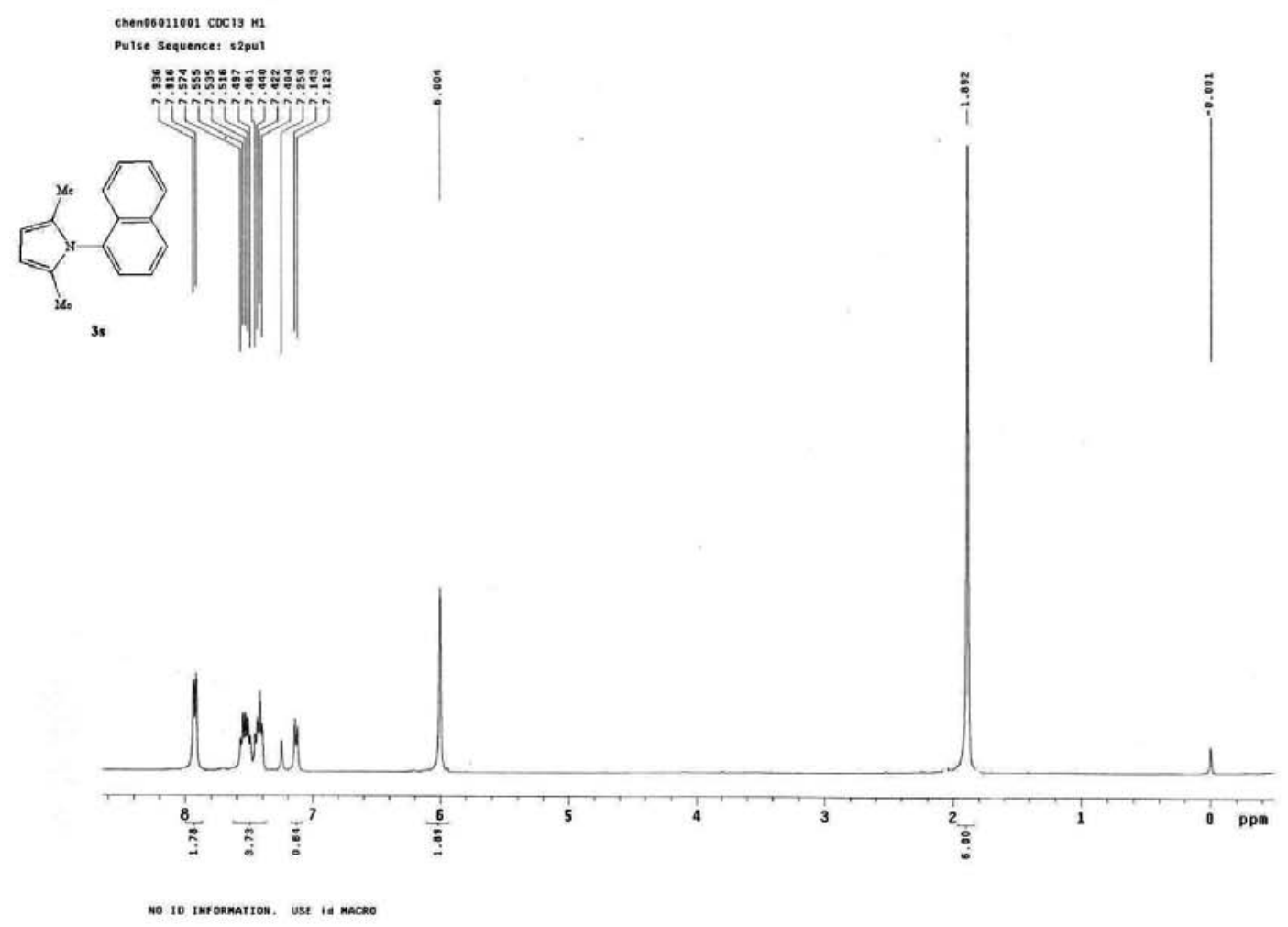

Chenosoliogr cocis c1s
Pulse sequencel spour

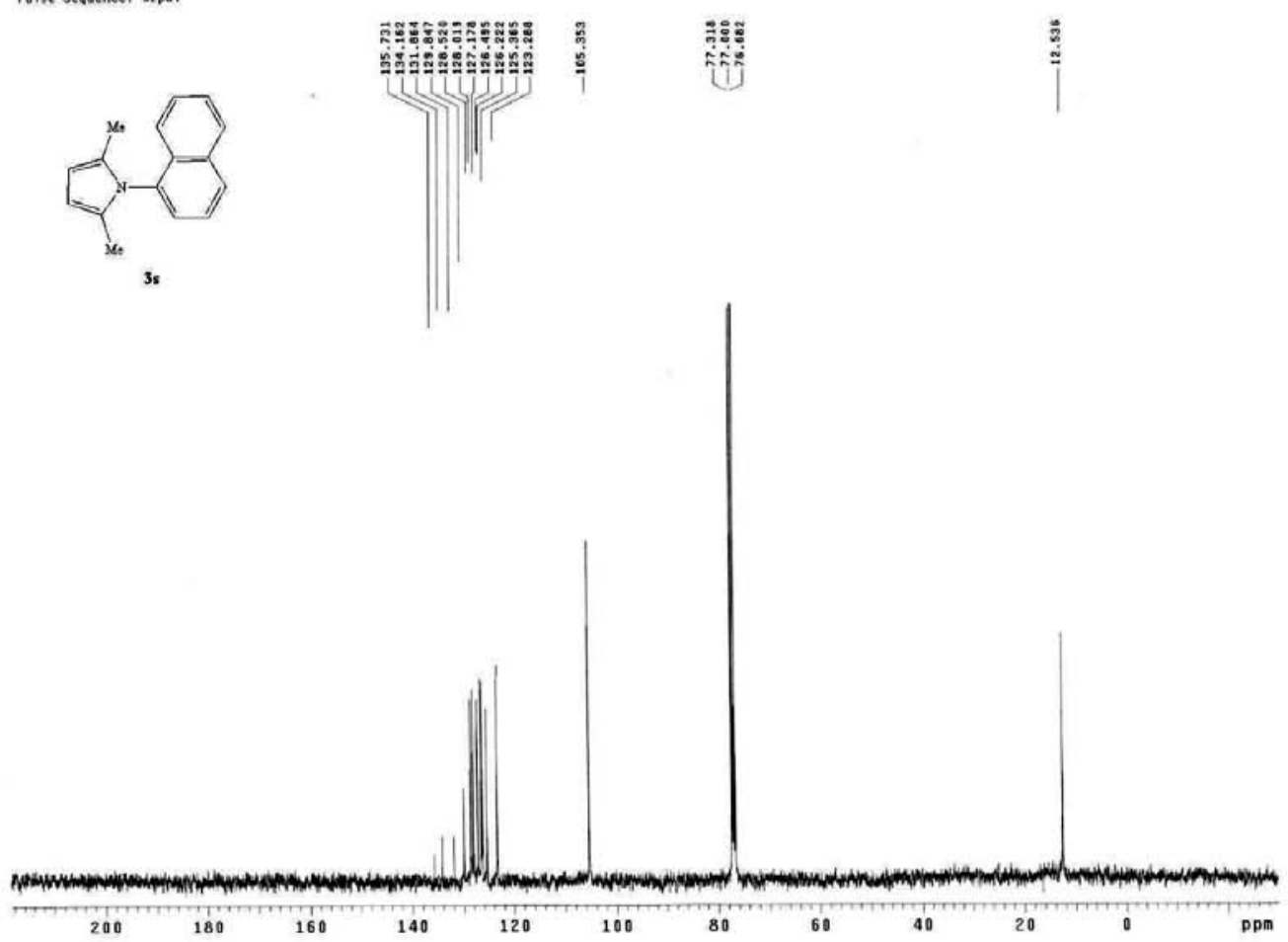

Figure S20. ${ }^{1} \mathrm{H}$ NMR of $3 \mathbf{s}\left(400 \mathrm{MHz}, \mathrm{CDCl}_{3}\right)$ and ${ }^{13} \mathrm{C} \mathrm{NMR}$ of $3 \mathbf{s}\left(100 \mathrm{MHz}, \mathrm{CDCl}_{3}\right)$. 


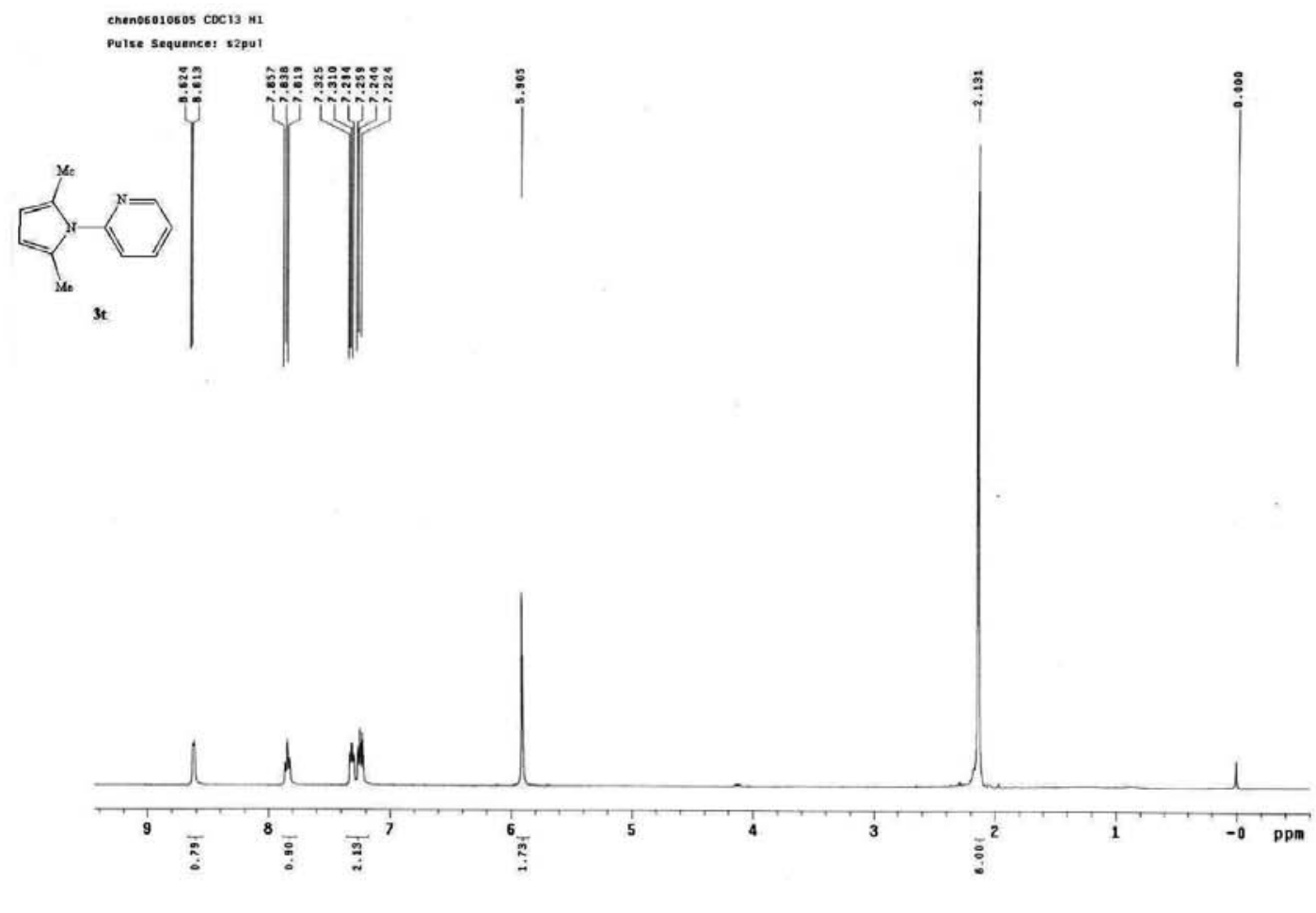

No Io Imrorkation. use ta racero

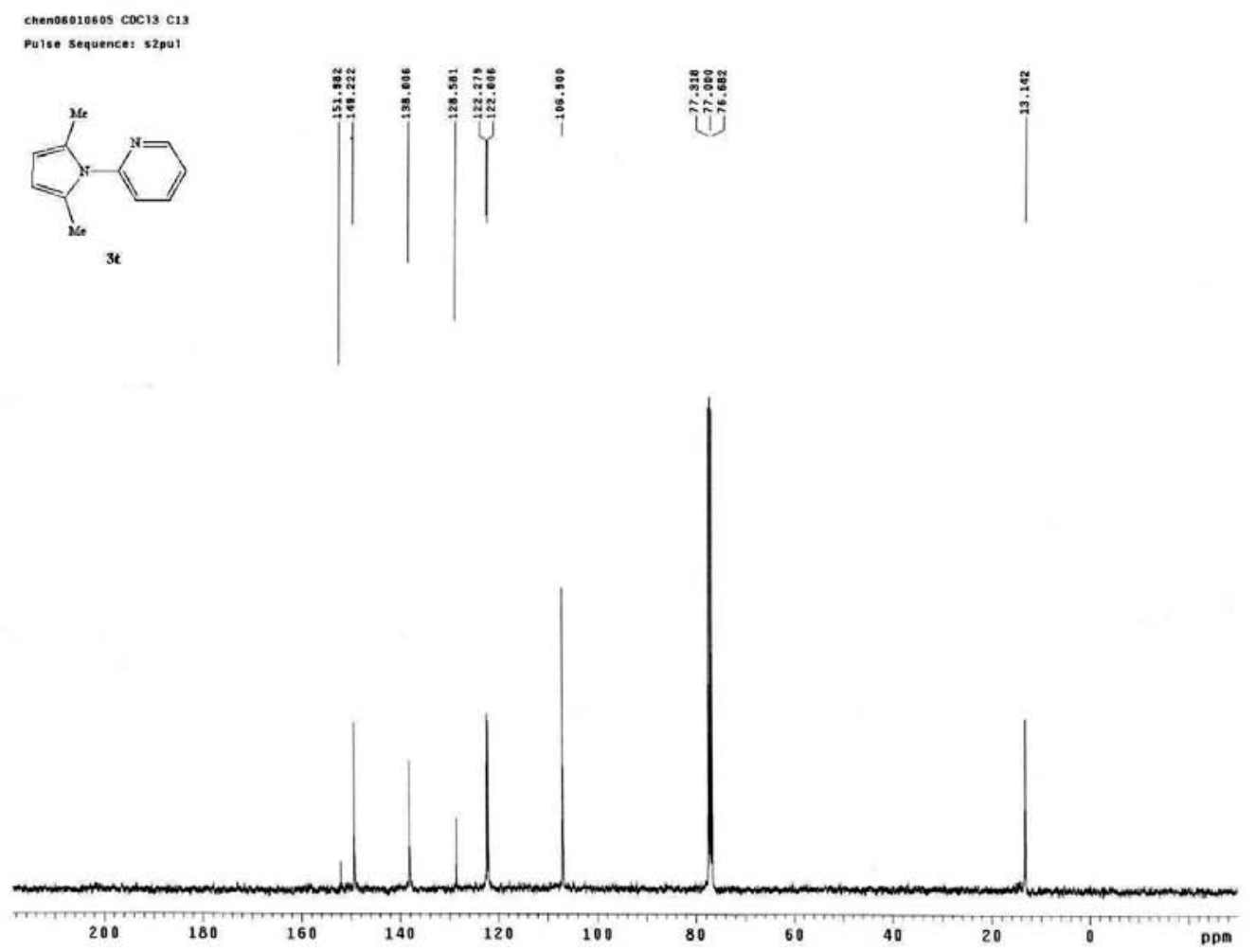

Figure S21. ${ }^{1} \mathrm{H}$ NMR of $\mathbf{3 t}\left(400 \mathrm{MHz}, \mathrm{CDCl}_{3}\right)$ and ${ }^{13} \mathrm{C} \mathrm{NMR}$ of $\mathbf{3 t}\left(100 \mathrm{MHz}, \mathrm{CDCl}_{3}\right)$. 
CJXOY $\mathrm{CDCl}^{3}$ "

Pulse Sequence: sâpul

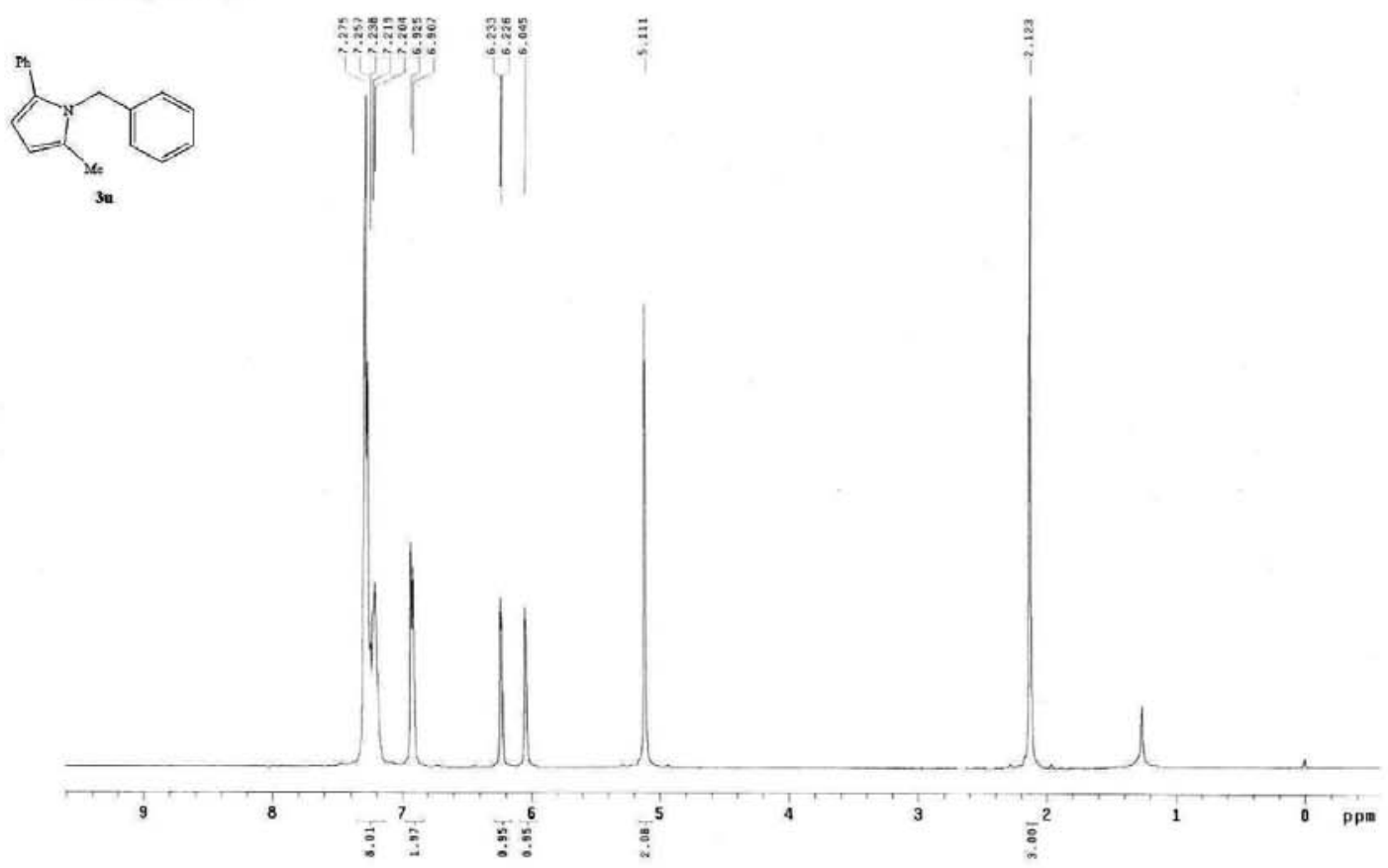

No Io furorkation. USE id macro

Caxor cac13 c13

pulse sequence: s2pul
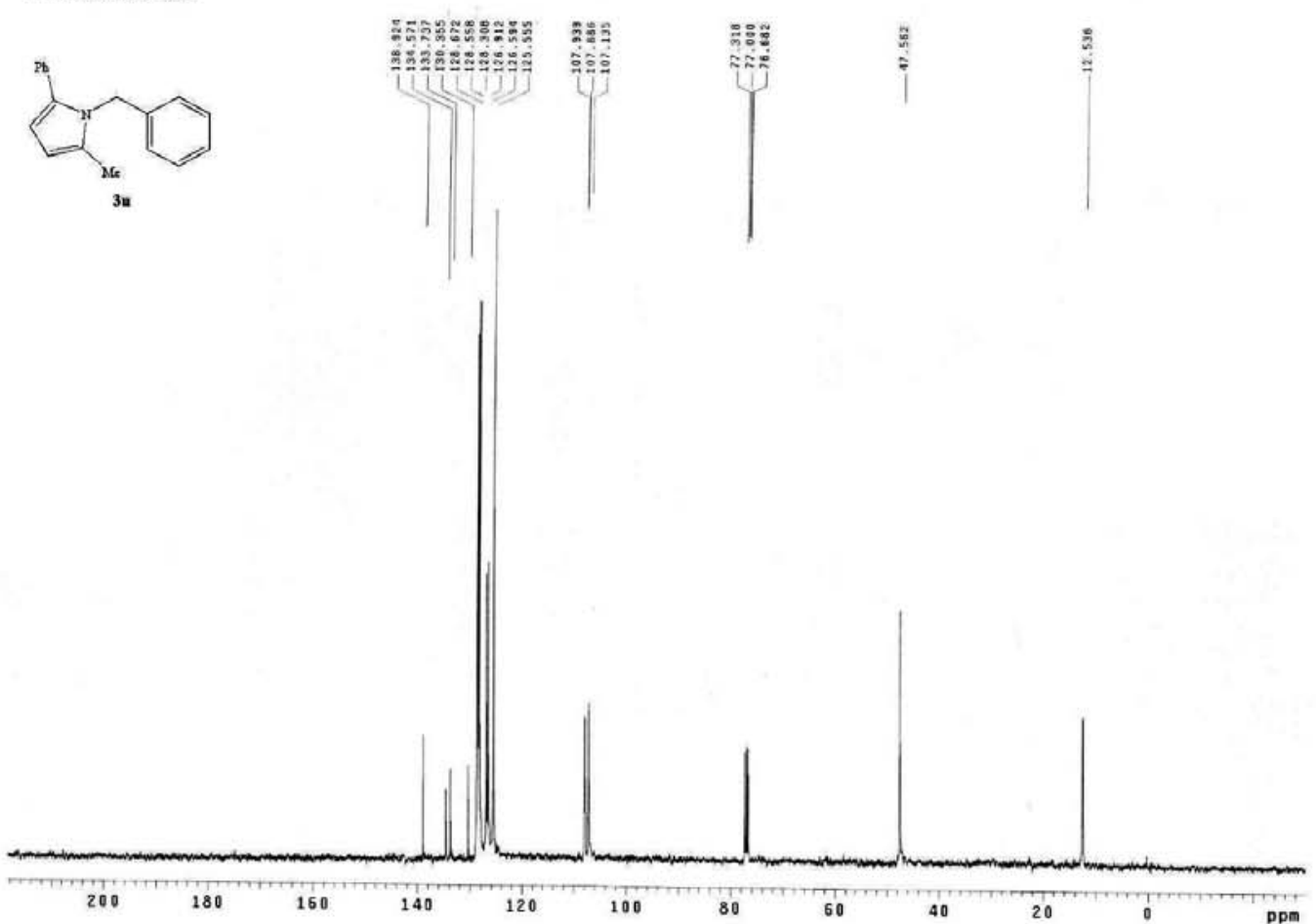

Figure S122. ${ }^{1} \mathrm{H}$ NMR of $\mathbf{3 u}\left(400 \mathrm{MHz}, \mathrm{CDCl}_{3}\right)$ and ${ }^{13} \mathrm{C} \mathrm{NMR}$ of $\mathbf{3 u}\left(100 \mathrm{MHz}, \mathrm{CDCl}_{3}\right)$. 


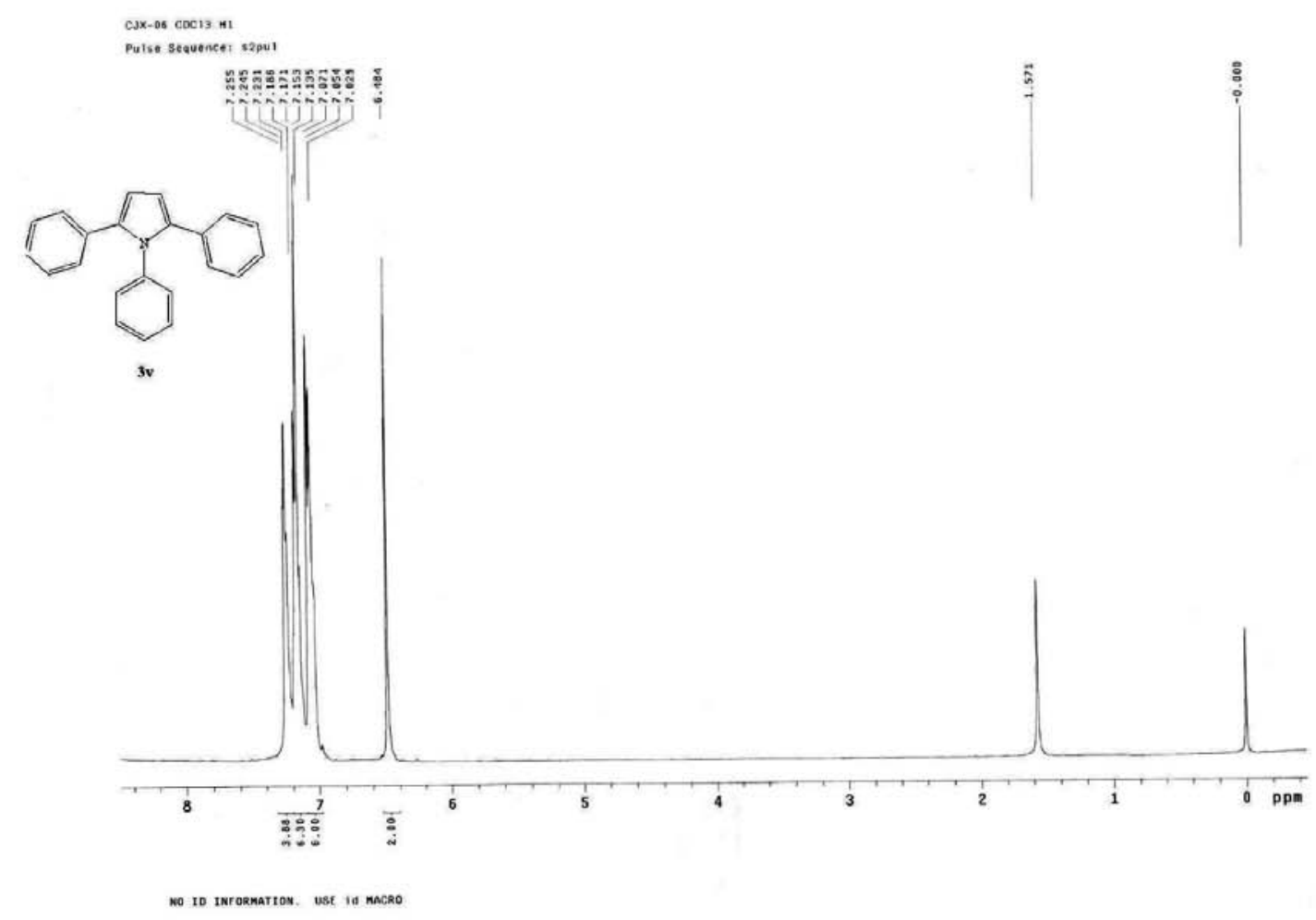

eJXony COC13 C19

Pulse sequences: szoul

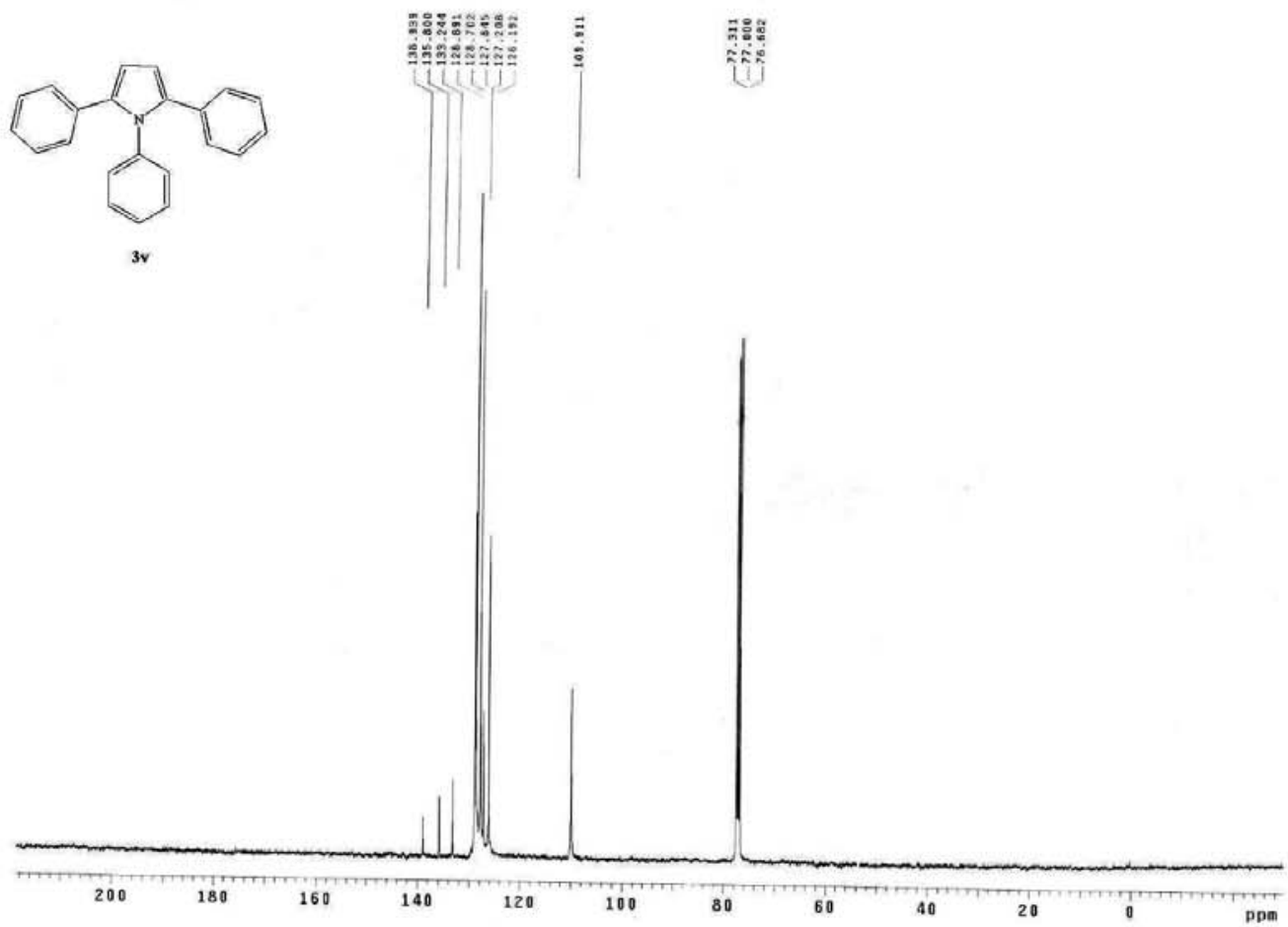

Figure S23. ${ }^{1} \mathrm{H}$ NMR of $\mathbf{3 v}\left(400 \mathrm{MHz}, \mathrm{CDCl}_{3}\right)$ and ${ }^{13} \mathrm{C} \mathrm{NMR}$ of $\mathbf{3 v}\left(100 \mathrm{MHz}, \mathrm{CDCl}_{3}\right)$. 


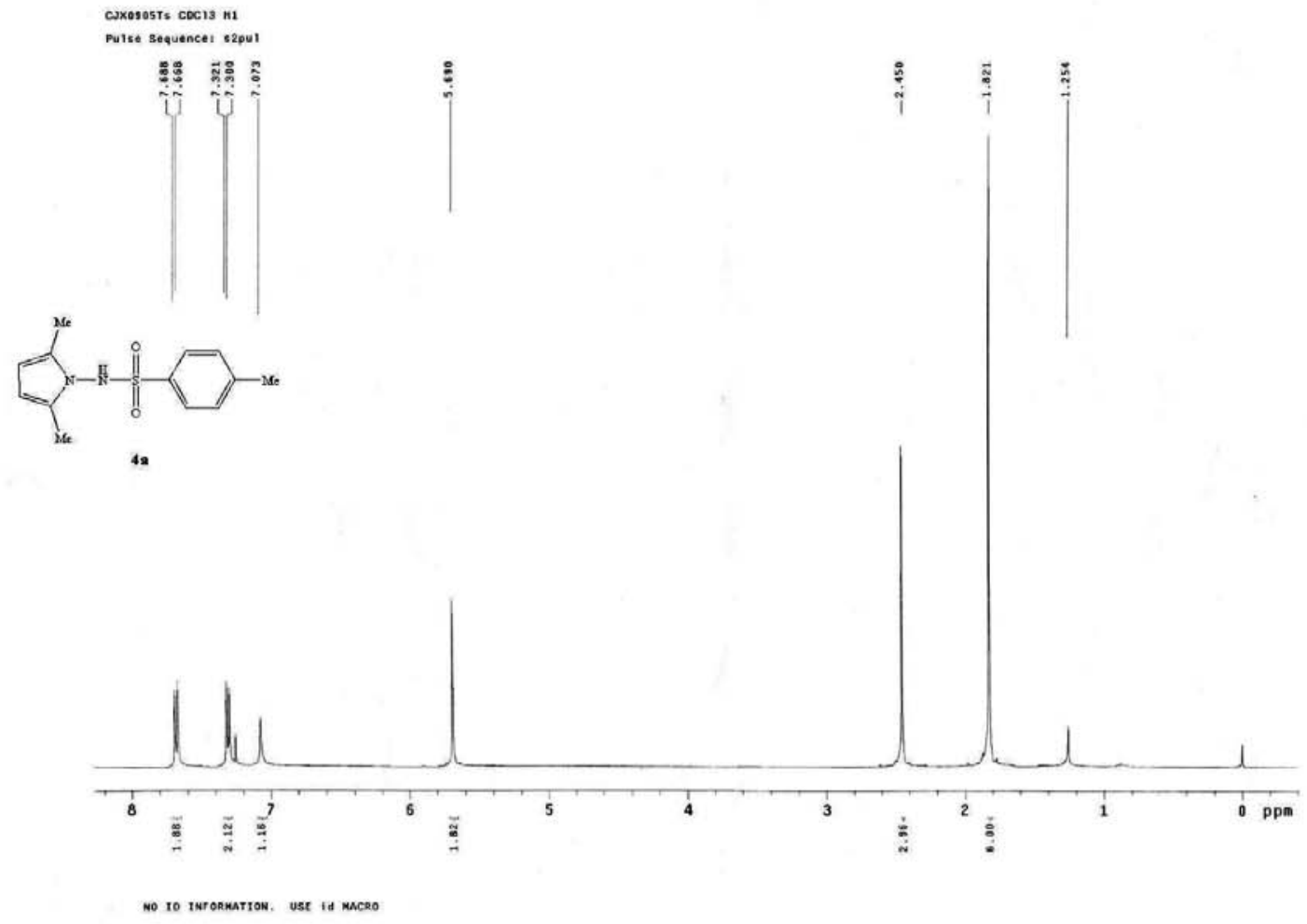

cuxososts Coc13 c13 Puise sequences sapur

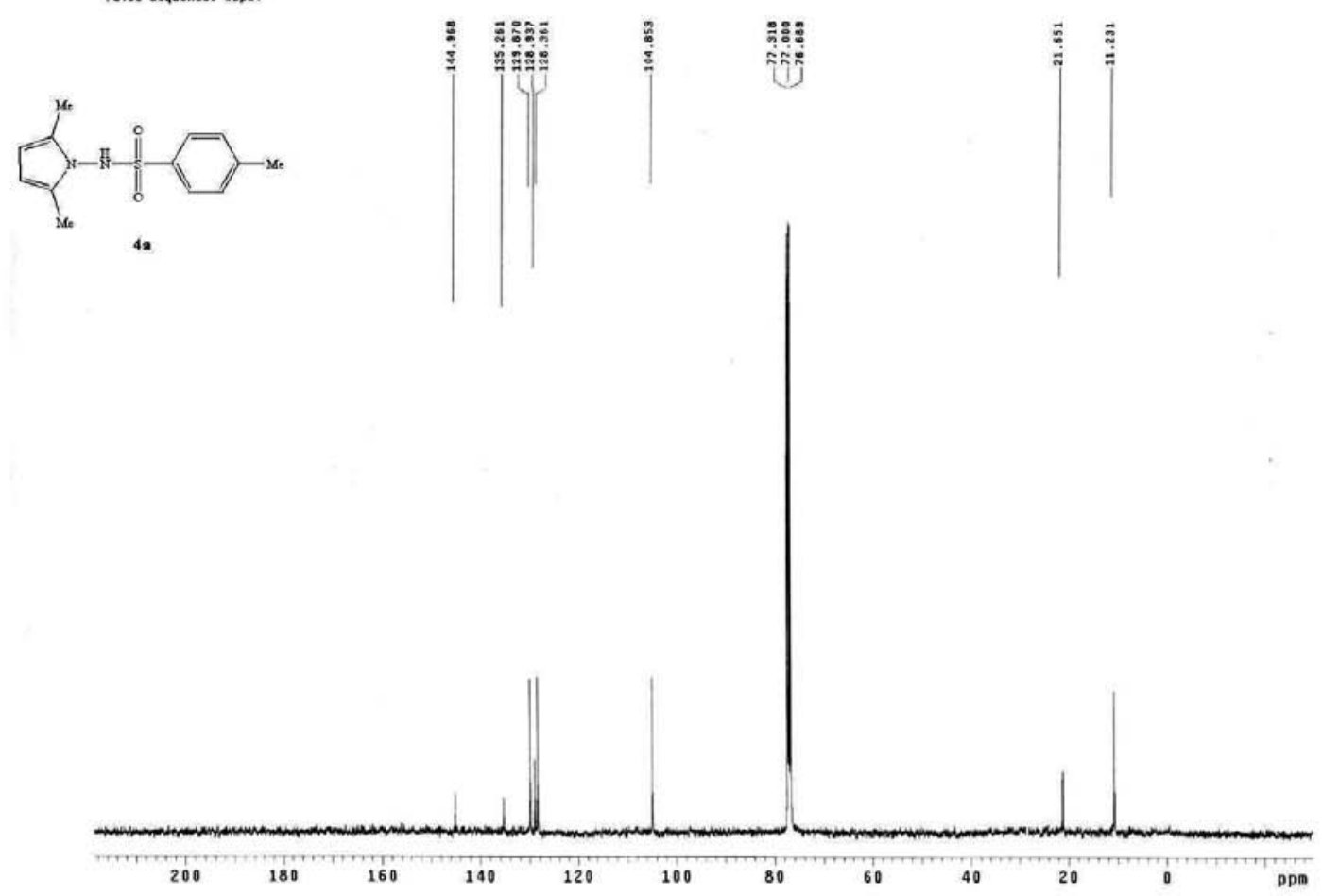

Figure S24. ${ }^{1} \mathrm{H}$ NMR of $4 \mathbf{a}\left(400 \mathrm{MHz}, \mathrm{CDCl}_{3}\right)$ and ${ }^{13} \mathrm{C} \mathrm{NMR}$ of $\mathbf{4 a}\left(100 \mathrm{MHz}, \mathrm{CDCl}_{3}\right)$. 